\title{
The spectral theorem for quaternionic unbounded normal operators based on the S-spectrum
}

Cite as: J. Math. Phys. 57, 023503 (2016); https://doi.org/10.1063/1.4940051

Submitted: 02 January 2015 . Accepted: 04 January 2016 . Published Online: 20 January 2016

Daniel Alpay, Fabrizio Colombo, and David P. Kimsey
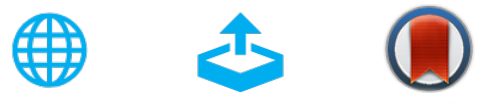

View Online

\section{ARTICLES YOU MAY BE INTERESTED IN}

Borel functional calculus for quaternionic normal operators

Journal of Mathematical Physics 58, 053501 (2017); https://

doi.org/10.1063/1.4982047

On the polar decomposition of right linear operators in quaternionic Hilbert spaces Journal of Mathematical Physics 57, 043502 (2016); https://

doi.org/10.1063/1.4945314

Foundations of Quaternion Quantum Mechanics

Journal of Mathematical Physics 3, 207 (1962); https://doi.org/10.1063/1.1703794

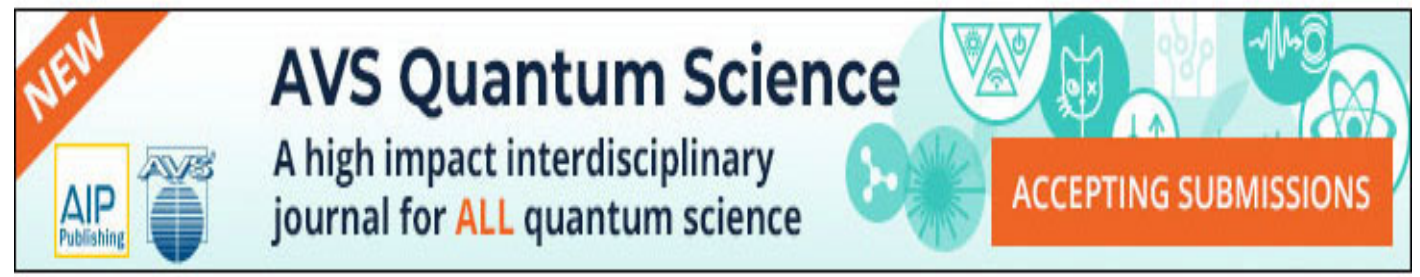




\title{
The spectral theorem for quaternionic unbounded normal operators based on the S-spectrum
}

\author{
Daniel Alpay, ${ }^{1, a)}$ Fabrizio Colombo, $\left.2, b\right)$ and David P. Kimsey ${ }^{1, c)}$ \\ ${ }^{1}$ Department of Mathematics, Ben-Gurion University of the Negev, Beer-Sheva 84105, Israel \\ ${ }^{2}$ Politecnico di Milano, Dipartimento di Matematica, Via E. Bonardi, 9, 20133 Milano, Italy
}

(Received 2 January 2015; accepted 4 January 2016; published online 20 January 2016)

\begin{abstract}
In this paper we prove the spectral theorem for quaternionic unbounded normal operators using the notion of $S$-spectrum. The proof technique consists of first establishing a spectral theorem for quaternionic bounded normal operators and then using a transformation which maps a quaternionic unbounded normal operator to a quaternionic bounded normal operator. With this paper we complete the foundation of spectral analysis of quaternionic operators. The $S$-spectrum has been introduced to define the quaternionic functional calculus but it turns out to be the correct object also for the spectral theorem for quaternionic normal operators. The lack of a suitable notion of spectrum was a major obstruction to fully understand the spectral theorem for quaternionic normal operators. A prime motivation for studying the spectral theorem for quaternionic unbounded normal operators is given by the subclass of unbounded antiself adjoint quaternionic operators which play a crucial role in the quaternionic quantum mechanics. @ 2016 AIP Publishing LLC. [http://dx.doi.org/10.1063/1.4940051]
\end{abstract}

\section{INTRODUCTION}

In Ref. 6, a spectral theorem for quaternionic unitary operators based on the $S$-spectrum was proved using an extension of Herglotz's theorem to the quaternions. ${ }^{7}$ In this paper, inspired by Ref. 6 , we treat the more general case of unbounded normal quaternionic operators.

The interest in spectral theory for quaternionic operators is motivated by the celebrated paper of Birkhoff and von Neumann, see Ref. 14, who showed that Schrödinger equation can be written only in the complex or quaternionic setting. Several authors have given important contributions to the development of the quaternionic version of quantum mechanics, see Refs. 2, 21, 23, and 28, but a correct notion of spectrum for quaternionic operators was still missing until the introduction of the $S$-spectrum, see, e.g., Ref. 18. As it is well known, in the classical formulation of quantum mechanics the spectral theory of unbounded self-adjoint operators plays a crucial role. In the fundamental paper, ${ }^{31}$ von Neumann used the spectral theorem for unitary operators to prove the spectral theorem for unbounded self-adjoint operators. In quaternionic quantum mechanics, the most important quaternionic operators are unbounded anti self-adjoint operators; these are a particular case of quaternionic unbounded normal operators treated in this paper.

Our strategy to prove the spectral theorem is as follows: first we deduce the spectral theorem for quaternionic bounded normal operators. The proof is based on a continuous functional calculus defined in Ref. 24 and a classical version of the Riesz representation theorem. After we establish a spectral theorem for quaternionic bounded normal operators, we deduce a spectral theorem for quaternionic unbounded normal operators from the bounded case and from a suitable transformation.

With the quaternionic spectral theorem based on the $S$-spectrum we complete the foundation of the quaternionic spectral theory that started some years ago with the introduction of the $S$-functional

\footnotetext{
a)E-mail address: dany@math.bgu.ac.il

b) E-mail address: fabrizio.colombo@polimi.it

c) E-mail address: dpkimsey@ gmail.com
} 
calculus. In fact, using the notion of slice hyperholomorphic functions, see Ref. 18, and the $S$-spectrum it is possible to define the quaternionic version of the Riesz-Dunford functional calculus which we now call quaternionic functional calculus or $S$-functional calculus.

We give a quick explanation of the reason why a consistent spectral theory for quaternionic operators is not so obvious. For simplicity consider a complex bounded operator $A: \mathcal{X} \rightarrow \mathcal{X}$ on a complex Banach space $\mathcal{X}$. The spectrum of $A$ is defined as

$$
\sigma(A)=\left\{\lambda \in \mathbb{C}: \lambda I_{\mathcal{X}}-A \text { is not invertible } B(\mathcal{X})\right\},
$$

where $B(\mathcal{X})$ denotes the Banach space of all bounded linear operators on $\mathcal{X}$. Given a normal (bounded) linear operator $T$ on a complex Hilbert space, in the spectral theorem

$$
T=\int_{\sigma(T)} \lambda d E(\lambda)
$$

the unique spectral measure $E(\lambda)$ associated to $T$ is supported on $\sigma(T)$, see, e.g., Ref. 19. The above notion of spectrum also appears in the Riesz-Dunford functional calculus, see Ref. 20, which is based on the Cauchy formula of holomorphic functions in which the Cauchy kernel is replaced by the resolvent operator $\left(\lambda I_{X}-T\right)^{-1}$. Taking a holomorphic function $h$ defined on an open set that contains the spectrum, we can use the Cauchy formula to define the linear operator $h(T)$.

From a historical viewpoint, a first attempt to generalize the classical notion of spectrum to quaternionic linear operators was to readapt the definition. To see the inconsistencies that occur, consider a right linear quaternionic operator $T: \mathcal{V} \rightarrow \mathcal{V}$ acting on a quaternionic two-sided Banach space $\mathcal{V}$. The symbol $\mathcal{B}(\mathcal{V})$ denotes the Banach space of all bounded right linear quaternionic operators on $\mathcal{V}$. The left spectrum $\sigma_{L}(T)$ of $T$ is related to the left-resolvent operator $\left(s I_{\mathcal{V}}-T\right)^{-1}$, i.e.,

$$
\sigma_{L}(T)=\left\{\mathrm{s} \in \mathbb{H}: s I_{\mathcal{V}}-T \text { is not invertible in } \mathcal{B}(\mathcal{V})\right\},
$$

where

$$
\left(s I_{\mathcal{V}}\right)(v)=s v, \quad v \in \mathcal{V} .
$$

The right spectrum $\sigma_{R}(T)$ of $T$ is associated with the right eigenvalue problem, i.e., the search for those quaternionis $s$ such that there exists a nonzero vector $v$ satisfying $T v=v s$. Observe that the operator $I_{\mathcal{V}} s-T$ associated with the right eigenvalue problem is not linear. Consequently, it is not clear what the resolvent operator ought to be. The quaternionic left-resolvent operator $\left(s I_{\mathcal{V}}-T\right)^{-1}$, as far as we know, is not hyperholomorphic in any sense. Consequently, the left-resolvent operator is not useful to define a hyperholomorphic quaternionic functional calculus. When we consider the right spectrum we just have the notion of eigenvalues. The above discussion shows that there is a problem in adapting the classical notion of spectrum to either the left or right quaternionic spectrum.

As we shall see, relative to obtaining a spectral theorem for quaternionic normal operators, the appropriate notion of spectrum is a new notion of spectrum which is as follows. The $S$-spectrum for a bounded linear operator $T$, see Ref. 18, is defined as

$$
\sigma_{S}(T)=\left\{\mathrm{s} \in \mathbb{H}: T^{2}-2 \operatorname{Re}(s) T+|s|^{2} I_{\mathcal{V}} \text { is not invertible in } \mathcal{B}(\mathcal{V})\right\},
$$

where $s=s_{0}+s_{1} e_{1}+s_{2} e_{2}+s_{3} e_{3}$ is a quaternion, $\left\{1, e_{1}, e_{2}, e_{3}\right\}$ is the standard basis of $\mathbb{H}, \operatorname{Re}(s)=s_{0}$ is the real part and the norm $|s|^{2}=s_{0}^{2}+s_{1}^{2}+s_{2}^{2}+s_{3}^{2}$, and the $S$-resolvent set is defined as

$$
\rho_{S}(T)=\mathbb{H} \backslash \sigma_{S}(T) \text {. }
$$

We are now ready to illustrate our main result, the spectral theorem for normal quaternionic operators. In this introduction we limit the discussion to the case of bounded normal operators but the theorem holds also for unbounded operators, see Theorem 6.2.

Consider the complex plane $\mathbb{C}_{j}:=\mathbb{R}+j \mathbb{R}$, for $j \in \mathbb{S}$, where $\mathbb{S}$ is the unit sphere of purely imaginary quaternions. Let $\mathbb{C}_{j}^{+}$denote all $p \in \mathbb{C}_{j}$ with $\operatorname{Im}(p) \geq 0$. Observe that $\mathbb{C}_{j}$ can be identified with a complex plane since $j^{2}=-1$ for every $j \in \mathbb{S}$. If $T$ is a (bounded) right linear normal operator on a quaternionic Hilbert space and $j \in \mathbb{S}$, then there is a unique spectral measure $E$ on $\sigma_{S}(T) \cap \mathbb{C}_{j}^{+}$such that

$$
\langle T x, y\rangle=\int_{\sigma_{S}(T) \cap \mathbb{C}_{j}^{+}} \operatorname{Re}(\mathrm{p}) d\langle E(p) x, y\rangle+\int_{\sigma_{S}(T) \cap \mathbb{C}_{j}^{+}} \operatorname{Im}(\mathrm{p}) d\langle J E(p) x, y\rangle,
$$


where $J$ is an anti-self adjoint and unitary operator which commutes with the spectral measure $E$. To show the deep difference between the complex spectral theory and the quaternionic spectral theory, we recall the quaternionic version of the Riesz-Dunford functional calculus, which suggests the notion of $S$-spectrum, see Refs. 16 and 15. This calculus involves two resolvent operators, namely, a left and right $S$-resolvent operator given by

$$
S_{L}^{-1}(s, T):=-\left(T^{2}-2 \operatorname{Re}(s) T+|s|^{2} I_{\mathcal{V}}\right)^{-1}\left(T-\bar{s} I_{\mathcal{V}}\right), \quad s \in \rho_{S}(T)
$$

and

$$
S_{R}^{-1}(s, T):=-\left(T-\bar{s} I_{\mathcal{V}}\right)\left(T^{2}-2 \operatorname{Re}(s) T+|s|^{2} I_{\mathcal{V}}\right)^{-1}, \quad s \in \rho_{S}(T),
$$

where $T \in \mathcal{B}(\mathcal{V})$ and $\rho_{S}(T)=\mathbb{H} \backslash \sigma_{S}(T)$ is the $S$-resolvent set. As one can see, the $S$-spectrum is suggested by the $S$-resolvent operators.

Let $\Omega \subset \mathbb{H}$ be a suitable domain that contains the $S$-spectrum of $T$. We define the quaternionic functional calculus for left slice hyperholomorphic functions $f: \Omega \rightarrow \mathbb{H}$ as

$$
f(T)=\frac{1}{2 \pi} \int_{\partial\left(\Omega \cap \mathbb{C}_{j}\right)} S_{L}^{-1}(s, T) d s_{j} f(s),
$$

where $d s_{j}=-d s j$; for right slice hyperholomorphic functions, we define

$$
f(T)=\frac{1}{2 \pi} \int_{\partial\left(\Omega \cap \mathbb{C}_{j}\right)} f(s) d s_{j} S_{R}^{-1}(s, T) .
$$

These definitions are well posed since the integrals depend neither on the open set $\Omega$ nor on the complex plane $\mathbb{C}_{j}$. Moreover, the resolvent equation, see Ref. 5, involves both $S$-resolvent operators, for $s$ and $p \in \rho_{S}(T)$ we have

$S_{R}^{-1}(s, T) S_{L}^{-1}(p, T)=\left\{\left(S_{R}^{-1}(s, T)-S_{L}^{-1}(p, T)\right) p-\bar{s}\left(S_{R}^{-1}(s, T)-S_{L}^{-1}(p, T)\right)\right\}\left(p^{2}-2 s_{0} p+|s|^{2}\right)^{-1}$.

Even though there are deep differences with respect to the classical resolvent equation for complex operators, all of the results that hold for the Riesz-Dunford functional calculus also hold for the quaternionic functional calculus. We now claim that to replace the complex spectral theory with the quaternionic spectral theory we have to replace the classical spectrum with the S-spectrum.

We conclude with some final remarks. In the case $T$ is a right linear operator on a finite-dimensional Hilbert space, the $S$-spectrum of $T$ coincides with the set of right eigenvalues of $T$; in the general case of a linear operator, the point $S$-spectrum coincides with the set of right eigenvalues. In the literature, the spectral theorem for quaternionic normal matrices based on the right spectrum is proved in Ref. 22 and there are some papers on the quaternionic spectral theorem, see, e.g., Refs. 23 and 34-36. However, the notion of spectrum in the papers ${ }^{23,34,36}$ is not made clear. In Ref. 25, a spectral theorem based on $S$-spectrum is proved for compact normal operators on a quaternionic Hilbert space. We point out that the $S$-resolvent operators are also used in Schur analysis in the realization of Schur functions in the slice hyperholomorphic setting see Ref. 1 and 8-10 and Refs. 3 and 12 for the classical case. In Refs. 11, 17, and 26 the problem of the generation of quaternionic groups and semigroups is treated using the $S$-spectrum. Functions of the generators of quaternionic groups of operators have been studied in Ref. 4.

The plan of the paper is as follows: In Section II we give some preliminaries; in Section III we recall a continuous functional calculus for bounded normal operators; in Section IV we prove the spectral theorem for bounded normal operators based on the $S$-spectrum; in Section V we introduce spectral integrals; finally, in Section VI we prove the spectral theorem for unbounded normal operators based on the $S$-spectrum.

\section{PRELIMINARIES}

Let $\mathcal{H}$ be a right linear quaternionic Hilbert space (see, e.g., Subsection 2.2 in Ref. 24), endowed with an $\mathbb{H}$-valued inner product $\langle\cdot, \cdot\rangle$ which satisfies, for every $\alpha, \beta \in \mathbb{H}$, and $x, y, z \in \mathcal{H}$, the following 
relations:

$$
\begin{aligned}
\langle x, y\rangle & =\overline{\langle y, x\rangle}, \\
\langle x, x\rangle & \geq 0 \quad \text { and }\|x\|^{2}:=\langle x, x\rangle=0 \Longleftrightarrow x=0, \\
\langle x \alpha+y \beta, z\rangle & =\langle x, z\rangle \alpha+\langle y, z\rangle \beta, \\
\langle x, y \alpha+z \beta\rangle & =\bar{\alpha}\langle x, y\rangle+\bar{\beta}\langle x, z\rangle .
\end{aligned}
$$

We call an operator $T: \mathcal{D}(T) \rightarrow \mathcal{H}$ right linear if

$$
T(x \alpha+y \beta)=(T x) \alpha+(T y) \beta,
$$

for all $x, y$ in the domain of $T$, denoted by $\mathcal{D}(T)$, and $\alpha, \beta \in \mathbb{H}$. The set of right linear operators on $\mathcal{H}$ will be denoted by $\mathcal{L}(\mathcal{H})$. Given $T \in \mathcal{L}(\mathcal{H})$ the range and kernel of $T$ will be given by

$$
\operatorname{Ran}(T)=\{\mathrm{y} \in \mathcal{H}: T x=y \text { for } x \in \mathcal{D}(T)\}
$$

and

$$
\operatorname{Ker}(T)=\{\mathrm{x} \in \mathcal{D}(T): T x=0\},
$$

respectively. We call an operator $T \in \mathcal{L}(\mathcal{H})$ bounded if

$$
\|T\|:=\sup _{\|x\| \leq 1}\|T x\|<\infty .
$$

In the sequel $\mathcal{B}(\mathcal{H})$ will denote the right Banach space of all bounded right linear operators on $\mathcal{H}$ endowed with the above norm.

Definition 2.1 We will call a subset $\mathcal{N} \subseteq \mathcal{H}$ an orthonormal basis if

$$
\begin{aligned}
\langle x, y\rangle & =0 \quad \text { for } x, y \in \mathcal{H} \text { so that } x \neq y, \\
\langle x, x\rangle & =1 \text { for } x \in \mathcal{H}, \\
\{x \in \mathcal{H}:\langle x, y\rangle \text { for all } y \in \mathcal{N}\} & =\{0\} .
\end{aligned}
$$

It can be checked in a similar manner to the classical complex Hilbert space case that every vector $x \in \mathcal{H}$ can be written as

$$
x=\sum_{y \in \mathcal{N}} y\langle x, y\rangle
$$

Lemma 2.2 Fix a right linear quaternionic Hilbert space $\mathcal{H}$. A right linear subspace $\mathcal{K}$ of $\mathcal{H} \oplus$ $\mathcal{H}$ satisfies

$$
\mathcal{K}=\{(x, T x): x \in \mathcal{D}(T)\},
$$

for some $T \in \mathcal{L}(\mathcal{H})$ if and only if

$$
(0, y) \in \mathcal{K} \Longrightarrow y=0
$$

Proof. If $\mathcal{K}$ is as in (2.5), then (2.6) follows directly from $T 0=0$. Conversely, if (2.6) holds, then $(x, y)$ and $(x, z)$ belonging to $\mathcal{K}$ implies that $y=z$, i.e., there exists a function $T: \mathcal{D}(T) \rightarrow \mathcal{H}$. The fact that $T \in \mathcal{L}(\mathcal{H})$ follows easily from the right linearity of $\mathcal{K}$. Thus, (2.5) holds.

Definition 2.3. An operator $T \in \mathcal{L}(\mathcal{H})$ is called closed if the set $\{(x, T x): x \in \mathcal{H}\}$ is a closed subset of $\mathcal{H} \times \mathcal{H}$. Let $S$ and $T$ both belong to $\mathcal{L}(\mathcal{H})$. We write $S=T$ if $\mathcal{D}(S)=\mathcal{D}(T)$ and $S x=T x$ for all $x \in \mathcal{D}(S)=\mathcal{D}(T)$. We write $S \subseteq T$ if $\mathcal{D}(S) \subseteq \mathcal{D}(T)$ and $S x=T x$ for all $x \in \mathcal{D}(S)$. Clearly, $S=T$ if and only if $S \subseteq T$ and $T \subseteq S$. An operator $T \in \mathcal{L}(\mathcal{H})$ is called closable if there exists a closed operator $U \in \mathcal{L}(\mathcal{H})$ so that $T \subseteq U$.

Theorem 2.4. Let $T \in \mathcal{L}(\mathcal{H})$. $T$ is closable if and only if

$$
\overline{\{(x, T x): x \in \mathcal{D}(T)\}}=\{(x, U x): \text { for some operator } U \in \mathcal{L}(\mathcal{H})\} \text {. }
$$


Proof. If $S \in \mathcal{L}(\mathcal{H})$ is any closed operator such that $T \subseteq S$, then

$$
\{(x, T x): x \in \mathcal{D}(T)\} \subseteq\{(x, S x): x \in \mathcal{D}(S)\} .
$$

Hence, as $S$ is closed,

$$
\overline{\{(x, T x): x \in \mathcal{D}(T)\}} \subseteq\{(x, S x): x \in \mathcal{D}(S)\} .
$$

Therefore, in view of Lemma 2.2, (2.7) holds.

Conversely, if (2.7) holds, then $T \subseteq U$ and hence $U$ is closed since

$$
\{(x, U x): x \in \mathcal{D}(U)\}
$$

is closed. Thus, $T$ is closable.

Definition 2.5. Let $T \in \mathcal{L}(\mathcal{H})$ be closable. We let

$$
\bar{T} x:=\lim _{n \uparrow \infty} T\left(x_{n}\right)
$$

denote the operator in $\mathcal{L}(\mathcal{H})$ with domain

$$
\mathcal{D}(\bar{T})=\left\{x \in \mathcal{H}: x=\lim _{n \uparrow \infty} x_{n} \text { for }\left\{x_{n}\right\}_{n=0}^{\infty} \subseteq \mathcal{D}(T) \text { and }\left\{T\left(x_{n}\right)\right\}_{n=0}^{\infty} \text { converges in } \mathcal{H}\right\} .
$$

In view of Theorem 2.4, the definition of $\bar{T}$ is independent of the choice of sequence $\left\{x_{n}\right\}_{n=0}^{\infty}$. Note that for any closed operator $U \in \mathcal{L}(\mathcal{H})$ such that $T \subseteq U$,

$$
\bar{T} \subseteq U \text {. }
$$

Definition 2.6. Given $T \in \mathcal{L}(\mathcal{H})$ which is densely defined, we let $T^{*} \in \mathcal{L}(\mathcal{H})$ denote the unique operator so that

$$
\langle T x, y\rangle=\left\langle x, T^{*} y\right\rangle, \quad x \in \mathcal{D}(T)
$$

The domain of $T^{*}$ is given by

$$
\mathcal{D}\left(T^{*}\right)=\{\mathrm{y} \in \mathcal{H}: \text { there exists } z \in \mathcal{H} \text { with }\langle T x, y\rangle=\langle x, z\rangle \text { for every } x \in \mathcal{D}(T)\} .
$$

Theorem 2.7. If $T \in \mathcal{L}(\mathcal{H})$ is densely defined and $W \in \mathcal{L}(\mathcal{H})$, then

(i) $T^{*} \in \mathcal{L}(\mathcal{H})$ is closed.

(ii) $\operatorname{Ran}(T)^{\perp}=\operatorname{Ker}\left(T^{*}\right)$.

(iii) If $T \subseteq W$, then $W^{*} \subseteq T^{*}$.

Proof. The proofs can completed in much the same way as the case when $\mathcal{H}$ is a complex Hilbert space (see, e.g., Proposition 1.6 in Ref. 33).

Theorem 2.8. If $T \in \mathcal{L}(\mathcal{H})$ is densely defined, then

(i) $T$ is closable if and only if $\mathcal{D}\left(T^{*}\right)$ is dense in $\mathcal{H}$.

(ii) If $T$ is closable, then $\bar{T}=T^{* *}$.

(iii) $T$ is closed if and only if $\bar{T}=T^{* *}$.

(iv) If $T$ is closable and $\operatorname{Ker}(T)=\{0\}$, then $T^{-1}$ is closable if and only if $\operatorname{Ker}(\bar{T})=\{0\}$. Moreover,

$$
(\bar{T})^{-1}=\overline{T^{-1}} \text {. }
$$

Proof. The proofs can completed in much the same way as the case when $\mathcal{H}$ is a complex Hilbert space (see, e.g., Theorem 1.8 in Ref. 33).

Definition 2.9. Let $T \in \mathcal{L}(\mathcal{H})$. We call $T$ normal if $T$ is densely defined, $T$ is closed, $\mathcal{D}(T)=$ $\mathcal{D}\left(T^{*}\right)$ and $T T^{*}=T^{*} T$.

Lemma 2.10. Let $T \in \mathcal{L}(\mathcal{H})$ be normal. If $S \in \mathcal{L}(\mathcal{H})$ so that $T \subseteq S$ and $\mathcal{D}(S) \subseteq D\left(S^{*}\right)$, then $S=T$. 
Proof. If $T \subseteq S$, then $S^{*} \subseteq T^{*}$ and hence

$$
\mathcal{D}(T) \subseteq \mathcal{D}(S) \subseteq \mathcal{D}\left(S^{*}\right) \subseteq \mathcal{D}\left(T^{*}\right)=\mathcal{D}(T),
$$

i.e., $\mathcal{D}(S)=\mathcal{D}(T)$. Therefore, $S=T$.

Definition 2.11. Let $T \in \mathcal{L}(\mathcal{H})$. We call $T$ self-adjoint, anti self-adjoint and unitary if $T=T^{*}$, $T=-T^{*}$, and $T T^{*}=T^{*} T=I_{\mathcal{H}}$, respectively.

Definition 2.12. Let $T \in \mathcal{L}(\mathcal{H})$ be densely defined and let $\mathcal{R}_{s}(T): \mathcal{D}\left(T^{2}\right) \rightarrow \mathcal{H}$ be given by

$$
\mathcal{R}_{s}(T) x=\left\{T^{2}-2 \operatorname{Re}(s) T+|s|^{2} I_{\mathcal{H}}\right\} x, \quad x \in \mathcal{D}\left(T^{2}\right) .
$$

The $S$-resolvent set of $T$ is defined as follows:

$$
\begin{aligned}
\rho_{S}(T)= & \left\{\mathrm{s} \in \mathbb{H}: \operatorname{Ker}\left(\mathcal{R}_{s}(T)\right)=\{0\}, \operatorname{Ran}\left(\mathcal{R}_{s}(T)\right) \text { is dense in } \mathcal{H}\right. \text { and } \\
& \left.\mathcal{R}_{S}(T)^{-1} \in \mathcal{B}(\mathcal{H})\right\} .
\end{aligned}
$$

The $S$-spectrum is defined as

$$
\sigma_{S}(T)=\mathbb{H} \backslash \rho_{S}(T) .
$$

For bounded operators this definition is equivalent to the one given in the Introduction (see Ref. 24).

Theorem 2.13. Let $T \in \mathcal{B}(\mathcal{H})$. Then the $S$-spectrum is a compact non-empty subset of $\mathbb{H}$ and

$$
\sigma_{S}(T) \subseteq\{p \in \mathbb{H}: 0 \leq|p| \leq\|T\|\} .
$$

Proof. See Theorem 3.2.6 in Ref. 18.

Theorem 2.14. Let $T \in \mathcal{L}(\mathcal{H})$ be densely defined. If $p=p_{0}+i p_{1} \in \sigma_{S}(T)$ for $i \in \mathbb{S}$ and $p_{0}, p_{1} \in$ $\mathbb{R}$, then $p_{0}+j p_{1} \in \sigma_{S}(T)$ for all $j \in \mathbb{S}$.

Proof. The proof of the assertion follows directly from the definition of the $S$-spectrum. If $s \in$ $\sigma_{S}(T)$, then it follows immediately from the definition of $\sigma_{S}(T)$ that all the quaternions with the same real part and the same modulus belong to the $S$-spectrum of $T$.

Theorem 2.15. Let $T \in \mathcal{L}(\mathcal{H})$. The following statements hold:

(i) If $T$ is positive, then $\sigma_{S}(T) \subseteq[0, \infty)$. If, in particular, $T \in \mathcal{B}(\mathcal{H})$ is positive, then

$$
\sigma_{S}(T) \subseteq[0,\|T\|]
$$

(ii) If $T$ is self-adjoint, then $\sigma_{S}(T) \subseteq \mathbb{R}$. If, in particular, $T \in \mathcal{B}(\mathcal{H})$ is self-adjoint, then

$$
\sigma_{S}(T) \subseteq[-\|T\|,\|T\|] .
$$

(iii) If $T$ is anti self-adjoint, then $\sigma_{S}(T) \subseteq\{p \in \mathbb{H}: \operatorname{Re} p=0\}$. If, in particular, $T \in \mathcal{B}(\mathcal{H})$ is anti self-adjoint, then

$$
\sigma_{S}(T) \subseteq\{p \in \mathbb{H}: \operatorname{Re} p=0 \text { and }|p| \leq\|T\|\} .
$$

(iv) If $T$ is unitary, then $\sigma_{S}(T) \subseteq \mathbb{S}$.

Proof. If $T \in \mathcal{L}(\mathcal{H})$, then the containments illustrated in (i)-(iii) follow readily from the definition of $\sigma_{S}(T)$. If $T \in \mathcal{B}(\mathcal{H})$, then the containments illustrated in (i)-(iv) follow readily from (2.8).

We will also need the following two versions of the Riesz representation theorem.

Theorem 2.16. Let $X$ be a compact Hausdorff space and $\mathscr{C}(X, \mathbb{R})$ denote the normed space of real-valued continuous functions on $X$ together with the supremum norm $\|\cdot\|_{\infty}$. Corresponding to any bounded linear functional $\psi: \mathscr{C}(X, \mathbb{R}) \rightarrow \mathbb{R}$ there exists a signed Borel measure $\mu$ on $X$ such that

$$
\psi(f)=\int_{X} f(t) d \mu(t) \quad \text { for } \text { all } f \in \mathscr{C}(X, \mathbb{R})
$$


If, in addition, $\psi$ is a positive linear functional, then $\mu$ is a positive Borel measure on X. In both cases $\mu$ is unique.

Proof. See, e.g., Theorem D in Section 56 of Ref. 27 for the case when $\psi$ is a positive bounded linear functional on $X$ and, e.g., Chapter 21 in Ref. 32 for the more general case.

\section{A FUNCTIONAL CALCULUS FOR BOUNDED NORMAL OPERATORS}

Let $\mathcal{H}$ be a right quaternionic Hilbert space and $\mathcal{B}(\mathcal{H})$ denote the set of all bounded right linear operators on $\mathcal{H}$. In Ref. 24, established the existence of some functional calculi for a quaternionic bounded normal operator. Before introducing the functional calculus for bounded normal operators, we first need some notation and results. In the rest of the paper, we say quaternionic Hilbert space $\mathcal{H}$ for right quaternionic Hilbert space.

Theorem 3.1. Let $\mathcal{N}$ be an orthonormal basis of a quaternionic Hilbert space $\mathcal{H}$. Then every $x \in \mathcal{H}$ can be decomposed uniquely via

$$
x=\sum_{z \in \mathcal{N}} z\langle x, z\rangle
$$

where

$$
\sum_{z \in \mathcal{N}} z\langle x, z\rangle:=\sup \left\{\sum_{z \in \mathcal{N}_{f}} z\langle x, z\rangle: \mathcal{N}_{f} \text { is a non-empty finite subset of } \mathcal{N}\right\}
$$

Proof. See, e.g., Proposition 2.6 in Ref. 24 with the caveat that the inner product in Ref. 24 is antilinear in the first variable and linear in the second variable.

Definition 3.2. Let $J \in \mathcal{B}(\mathcal{H})$ be anti self-adjoint and unitary and $j \in \mathbb{S}$. Let $\mathcal{H}_{ \pm}^{j}$ denote the closed complex (with respect to the complex plane $\mathbb{C}_{j}$ ) subspaces given by

$$
\mathcal{H}_{ \pm}^{j}=\{x \in \mathcal{H}: J x= \pm x j\} .
$$

We will now formulate some useful results from Ref. 24 in the following lemma.

Lemma 3.3 (Ref. 24). If $J$ is an anti self-adjoint and unitary operator and $j \in \mathbb{S}$, then

(i) $\mathcal{H}_{ \pm}^{j} \neq\{0\}$.

(ii) As a $\mathbb{C}_{j}$-Hilbert space, $\mathcal{H}$ admits the following direct sum decomposition:

$$
\mathcal{H}=\mathcal{H}_{+}^{j} \oplus \mathcal{H}_{-}^{j} .
$$

Proof. The proofs of items (i) and (ii) can be found in Proposition 3.8(d) and Lemma 3.10 in Ref. 24, respectively.

Definition 3.4. Fix an orthonormal basis $\mathcal{N}$ of a quaternionic Hilbert space $\mathcal{H}$. The left scalar multiplication $L_{p}$ of $\mathcal{H}$ induced by $\mathcal{N}$ is the map

$$
(p, x) \in \mathbb{H} \times \mathcal{H} \mapsto p x \in \mathcal{H}
$$

given by

$$
p x:=\sum_{y \in \mathcal{N}} y p\langle x, y\rangle
$$

Lemma 3.5 (Statement (a) of Proposition 3.8 in Ref. 24). Let $\mathcal{H}$ be a quaternionic Hilbert space. If $J \in \mathcal{B}(\mathcal{H})$ is an anti self-adjoint and unitary operator, then corresponding to any fixed $j \in \mathbb{S}$, there exists a left-scalar multiplication $L_{p}$ so that

$$
J=L_{j} .
$$


In the following theorem, we will make use of the operator $|T|:=\left(T^{*} T\right)^{1 / 2}$ for $T \in \mathcal{B}(\mathcal{H})$. See Section 2.4 of Ref. 24 for a definition of the square root of a positive operator which relies on the functional calculus therein.

Theorem 3.6. Let $T \in \mathcal{B}(\mathcal{H})$ be normal. Then there exist uniquely determined operators $A:=$ $(1 / 2)\left(T+T^{*}\right)$ and $B:=(1 / 2)\left|T-T^{*}\right|$ which both belong to $\mathcal{B}(\mathcal{H})$ and an operator $J \in \mathcal{B}(\mathcal{H})$ which is uniquely determined on $\left\{\operatorname{Ker}\left(T-T^{*}\right)\right\}^{\perp}$ so that the following properties hold:

(i) $T=A+J B$.

(ii) $A$ is self-adjoint and $B$ is positive.

(iii) $J$ is anti self-adjoint and unitary.

(iv) $A, B$, and $J$ mutually commute.

(v) For any fixed $j \in \mathbb{S}$, there exists an orthonormal basis $\mathcal{N}_{j}$ of $\mathcal{H}$ with the property that $J=L_{j}$.

Proof. Properties (i)-(iv) appear in Theorem J on page 4 of Ref. 24. Property (v) follows from Lemma 3.5.

Definition 3.7. Let $\Omega \subseteq \mathbb{H}$. We call $\Omega$ axially symmetric if $p_{0}+i p_{1} \in \Omega$ with $p_{0}, p_{1} \in \mathbb{R}$ and $i \in \mathbb{S}$, then $p_{0}+j p_{1} \in \Omega \cap \mathbb{C}_{j}$ for all $j \in \mathbb{S}$.

Remark 3.8. Let $T \in \mathcal{L}(\mathcal{H})$. In view of Theorem $2.14, \sigma_{S}(T)$ is an axially symmetric subset of $\mathbb{H}$.

Definition 3.9. Let $\Omega \subseteq \mathbb{H}$ be an axially symmetric set and let $D \subseteq \mathbb{R}^{2}$ be such that

$$
D=\left\{(u, v) \in \mathbb{R}^{2}: u+j v \in \Omega \text { for some } j \in \mathbb{S}\right\} .
$$

Let $\mathcal{S}(\Omega, \mathbb{H})$ denote the quaternionic linear space of slice continuous functions, i.e., $\mathcal{S}(\Omega, \mathbb{H})$ consists of functions $f: \Omega \rightarrow \mathbb{H}$ of the form

$$
f(u+j v)=f_{0}(u, v)+j f_{1}(u, v) \quad \text { for }(u, v) \in D \text { and for } j \in \mathbb{S},
$$

where $f_{0}$ and $f_{1}$ are continuous $\mathbb{H}$-valued functions on $D$ so that

$$
f_{0}(u, v)=f_{0}(u,-v) \text { and } f_{1}(u, v)=-f_{1}(u,-v) .
$$

If $f_{0}$ and $f_{1}$ are real-valued, then we say that the continuous slice function $f$ is intrinsic. The subspace of intrinsic continuous slice functions is denoted by $\mathcal{S}_{\mathbb{R}}(\Omega, \mathbb{H})$.

Remark 3.10. We observe that if $f \in \mathcal{S}_{\mathbb{R}}(\Omega, \mathbb{H})$ and we consider the restriction of $f$ to $\Omega_{j}:=$ $\Omega \cap \mathbb{C}_{j}$, where $j \in \mathbb{S}$, then $f$ has values in $\mathbb{C}_{j}$. This fact makes clear the notation $\mathcal{S}_{\mathbb{R}}\left(\Omega_{j}, \mathbb{C}_{j}\right)$.

The following functional calculus will be useful for proving a spectral theorem for a normal operator $T \in \mathcal{B}(\mathcal{H})$.

Theorem 3.11 (Theorem 7.4 in Ref. 24). Let $T \in \mathcal{B}(\mathcal{H})$ be normal. There exists a unique continuous *-homomorphism

$$
\Psi_{\mathbb{R}, T}: f \in \mathcal{S}_{\mathbb{R}}\left(\sigma_{S}(T), \mathbb{H}\right) \mapsto f(T) \in \mathcal{B}(\mathcal{H})
$$

of real-Banach unital $C^{*}$-algebras such that

(i) $\Psi_{\mathbb{R}, T}\left(\chi_{\sigma_{S}(T)}\right)=I_{\mathcal{H}}$, where

$$
\chi_{\sigma_{S}(T)}(p)=\left\{\begin{array}{lll}
1 & \text { if } & p \in \sigma_{S}(T) \\
0 & \text { if } & p \notin \sigma_{S}(T)
\end{array} .\right.
$$

(ii) $\quad \Psi_{\mathbb{R}, T}(\mathrm{id})=T$, where id denotes the inclusion map from $\sigma_{S}(T)$ to $\mathbb{H}$.

(iii) If $J$ is as in Theorem 3.6, then $J$ commutes with the normal operator $f(T)$.

(iv) If $f \in \mathcal{S}_{\mathbb{R}}\left(\sigma_{S}(T), \mathbb{H}\right)$, then $\|f(T)\|=\|f\|_{\infty}$.

(v) If $f \in \mathcal{S}_{\mathbb{R}}\left(\sigma_{S}(T), \mathbb{H}\right)$, then

$$
\sigma_{S}(f(T))=f\left(\sigma_{S}(T)\right) .
$$


Remark 3.12. For the convenience of the reader, we will now outline the construction of $f(T)$ for $f \in \mathcal{S}_{\mathbb{R}}\left(\sigma_{S}(T), \mathbb{H}\right)$. Fix $j \in \mathbb{S}$. Since $\sigma_{S}(T) \cap \mathbb{C}_{j}$ is compact, there exist sequences of real-valued polynomials $\left\{\phi_{n}\right\}_{n=0}^{\infty}$ and $\left\{\psi_{n}\right\}_{n=0}^{\infty}$ on $D \subseteq \mathbb{R}^{2}$, where $D$ is as in Definition 3.9, with

$$
\phi_{n}(u, v)=\phi_{n}(u,-v) \text { for } n=0,1, \ldots
$$

and

$$
\psi_{n}(u, v)=-\psi_{n}(u,-v) \text { for } n=0,1, \ldots
$$

so that

$$
f_{0}(u, v)=\lim _{n \uparrow \infty} \phi_{n}(u, v) \text { uniformly on } D \subseteq \mathbb{R}^{2}
$$

and

$$
f_{1}(u, v)=\lim _{n \uparrow \infty} \psi_{n}(u, v) \text { uniformly on } D \subseteq \mathbb{R}^{2},
$$

respectively.

Since $\phi_{n}(u, v)$ and $\psi_{n}(u, v)$ have real coefficients and $A$ and $B$ are commuting self-adjoint operators, it follows easily that $\phi_{n}(A, B) \in \mathcal{B}(\mathcal{H})$ and $\psi_{n}(A, B) \in \mathcal{B}(\mathcal{H})$ are self-adjoint. Next, we define

$$
f_{0}(T) x:=\lim _{n \uparrow \infty} \phi_{n}(A, B) x, \quad x \in \mathcal{H}
$$

and

$$
f_{1}(T) x:=\lim _{n \uparrow \infty} \psi_{n}(A, B) x, \quad x \in \mathcal{H} .
$$

Note that the limit in (3.7) exists since $\phi_{n}(A, B)=\phi_{n}(A, B)^{*}$ and hence

$$
\begin{aligned}
\left\|\left\{\phi_{m}(A, B)-\phi_{n}(A, B)\right\} x\right\|^{2} & =\left\langle\left\{\phi_{m}(A, B)-\phi_{n}(A, B)\right\}^{2} x, x\right\rangle \\
& \leq\left\|\left\{\phi_{m}(A, B)-\phi_{n}(A, B)\right\}^{2}\right\|\|x\|^{2} \\
& =\left\|\phi_{m}-\phi_{n}\right\|_{\infty}^{2}\|x\|^{2} \\
& \rightarrow 0, \quad \text { as } \quad m, n \uparrow \infty,
\end{aligned}
$$

since (3.5) holds. Note that formula (7.3) in Ref. 24 was used to obtain (3.9). The verification of the existence of the limit given in (3.8) is similar. The normal operator $f(T) \in \mathcal{B}(\mathcal{H})$ is given by

$$
f(T)=f_{0}(T)+J f_{1}(T)
$$

Lemma 3.13. Fix a normal operator $T \in \mathcal{B}(\mathcal{H})$. If

$$
f=f_{0}+f_{1} j \in \mathcal{S}_{\mathbb{R}}\left(\sigma_{S}(T) \cap \mathbb{C}_{j}, \mathbb{H}\right),
$$

then $f_{0}(T)$ and $f_{1}(T)$ (given in (3.7) and (3.8), respectively) are self-adjoint.

Proof. We claim that

$$
\lim _{n \uparrow \infty}\left\langle\phi_{n}(A, B) x, y\right\rangle=\left\langle f_{0}(T) x, y\right\rangle, \quad x, y \in \mathcal{H},
$$

and

$$
\lim _{n \uparrow \infty}\left\langle\psi_{n}(A, B) x, y\right\rangle=\left\langle f_{1}(T) x, y\right\rangle, \quad x, y \in \mathcal{H} .
$$

Assertion (3.11) follows directly from

$$
\begin{aligned}
\left|\left\langle\phi_{n}(A, B) x, y\right\rangle-\left\langle f_{0}(T) x, y\right\rangle\right| & \leq\left\|\phi_{n}(A, B)-f_{0}(T)\right\|\|x\|\|y\| \\
& =\left\|\phi_{n}-f_{0}\right\|_{\infty}\|x\|\|y\|,
\end{aligned}
$$


where item (iv) of Theorem 3.11 was used to obtain the last line. Assertion (3.12) is shown in much the same way. In view of (3.11),

$$
\begin{aligned}
\left\langle f_{0}(T) x, y\right\rangle & =\lim _{n \uparrow \infty}\left\langle\phi_{n}(A, B) x, y\right\rangle \\
& =\lim _{n \uparrow \infty}\left\langle x, \phi_{n}(A, B) y\right\rangle \\
& =\left\langle x, f_{0}(T) y\right\rangle, \quad x, y \in \mathcal{H} .
\end{aligned}
$$

Thus, $f_{0}(T)$ is self-adjoint. The fact that $f_{1}(T)$ is self-adjoint can be completed in much the same way using (3.12).

\section{THE SPECTRAL THEOREM FOR BOUNDED NORMAL OPERATORS BASED ON THE S-SPECTRUM}

In this section we shall consider normal operators $T$ which are bounded, i.e., $T \in \mathcal{B}(\mathcal{H})$. We will generate a spectral theorem based on the $S$-spectrum using Theorems 2.16 and 3.11. This approach is analogous to a well-known approach in the classical case, i.e., when $\mathcal{H}$ is a complex Hilbert space. See, e.g., the book of $\operatorname{Lax}^{29}$ for details.

Fix a normal operator $T \in \mathcal{B}(\mathcal{H})$ and $j \in \mathbb{S}$. By Theorem 3.6, there exist commuting operators $A:=(1 / 2)\left(T+T^{*}\right)$ and $B:=(1 / 2)\left|T-T^{*}\right|$, where $|W|=\left(W^{*} W\right)^{1 / 2}$ for $W \in \mathcal{B}(\mathcal{H})$, and $J$ which all belong to $\mathcal{B}(\mathcal{H})$ so that $T=A+J B$ and $A$ and $B$ are uniquely determined by $T$.

Lemma 4.1. Let $\mathscr{C}\left(\Omega_{j}^{+}, \mathbb{R}\right)$ denote the set of real-valued continuous functions on $\Omega_{j}^{+}=\sigma_{S}(T) \cap$ $\mathbb{C}_{j}^{+}$and $\mathcal{S}_{\mathbb{R}}\left(\Omega_{j}, \mathbb{R}\right)$ denote the set of real-valued functions in $\mathcal{S}_{\mathbb{R}}\left(\Omega_{j}, \mathbb{H}\right)$, where $\Omega_{j}=\sigma_{S}(T) \cap \mathbb{C}_{j}$. Let $\mathscr{C}_{0}\left(\Omega_{j}^{+}, \mathbb{R}\right)$ denote the subset of functions $f \in \mathscr{C}\left(\Omega_{j}^{+}, \mathbb{R}\right)$ such that $\left.f\right|_{\Omega_{j} \cap \mathbb{R}}=0$. The following statements hold:

(i) There exists a bijection between $\mathscr{C}\left(\Omega_{j}^{+}, \mathbb{R}\right)$ and $\mathcal{S}_{\mathbb{R}}\left(\Omega_{j}, \mathbb{R}\right)$.

(ii) If $\Omega_{j}^{+} \cap \mathbb{R} \neq \emptyset$, then there exists a bijection between $\mathscr{C}_{0}\left(\Omega_{j}^{+}, \mathbb{R}\right)$ and purely imaginary functions in $\mathcal{S}_{\mathbb{R}}\left(\Omega_{j}, \mathbb{H}\right)$.

(iii) If $\Omega_{j}^{+} \cap \mathbb{R}=\emptyset$, then there exists a bijection between $\mathscr{C}\left(\Omega_{j}^{+}, \mathbb{R}\right)$ and purely imaginary functions in $\mathcal{S}_{\mathbb{R}}\left(\Omega_{j}, \mathbb{H}\right)$.

Proof. The proof is broken into steps.

Step 1: Prove (i).

If $g \in \mathscr{C}\left(\Omega_{j}^{+}, \mathbb{R}\right)$, then the function

$$
\tilde{g}(u, v)=\left\{\begin{array}{lll}
g(u, v) & \text { if } & u+j v \in \Omega_{j}^{+} \\
g(u,-v) & \text { if } & u+j v \in \Omega_{j}^{-}
\end{array},\right.
$$

where

$$
\Omega_{j}^{-}=\sigma_{S}(T) \cap \mathbb{C}_{j}^{-}
$$

and

$$
\mathbb{C}_{j}^{-}=\{u+j v: u \in \mathbb{R} \text { and } v \leq 0\}
$$

belongs to $\mathcal{S}_{\mathbb{R}}\left(\Omega_{j}, \mathbb{R}\right)$. Conversely, if $f \in \mathcal{S}_{\mathbb{R}}\left(\Omega_{j}, \mathbb{C}_{j}\right)$ is real-valued, then

$$
\tilde{f}=\left.f\right|_{\Omega_{j}^{+}} \in \mathscr{C}\left(\Omega_{j}^{+}, \mathbb{R}\right) .
$$

Step 2: Prove (ii).

If $\Omega_{j}^{+} \cap \mathbb{R} \neq \emptyset$ and $g \in \mathscr{C}_{0}\left(\Omega_{j}^{+}, \mathbb{R}\right)$, then the function

$$
\tilde{g}(u, v)=\left\{\begin{array}{lll}
j g(u, v) & \text { if } & u+j v \in \Omega_{j}^{+} \\
-j g(u,-v) & \text { if } & u+j v \in \Omega_{j}^{-}
\end{array}\right.
$$


belongs to $\mathcal{S}_{\mathbb{R}}\left(\Omega_{j}, \mathbb{C}_{j}\right)$. Conversely, if $f \in \mathcal{S}_{\mathbb{R}}\left(\Omega_{j}, \mathbb{C}_{j}\right)$ is purely imaginary, i.e., $f=j f_{1}$, with $f_{1}$ realvalued, then $\tilde{f}=\left.j f\right|_{\Omega_{j}^{+}} \in \mathscr{C}_{0}\left(\Omega_{j}^{+}, \mathbb{R}\right)$.

Step 3: Prove (iii).

If $\Omega_{j}^{+} \cap \mathbb{R}=\emptyset$ and $g \in \mathscr{C}\left(\Omega_{j}^{+}, \mathbb{R}\right)$, then the function

$$
\tilde{g}(u, v)=\left\{\begin{array}{lll}
j g(u, v) & \text { if } & u+j v \in \Omega_{j}^{+} \\
-j g(u,-v) & \text { if } & u+j v \in \Omega_{j}^{-}
\end{array}\right.
$$

belongs to $\mathcal{S}_{\mathbb{R}}\left(\Omega_{j}, \mathbb{C}_{j}\right)$. Conversely, if $f \in \mathcal{S}_{\mathbb{R}}\left(\Omega_{j}, \mathbb{C}_{j}\right)$ is purely imaginary, i.e., $f=j f_{1}$, with $f_{1}$ realvalued, then $\tilde{f}=\left.j f\right|_{\Omega_{j}^{+}} \in \mathscr{C}\left(\Omega_{j}^{+}, \mathbb{R}\right)$.

Let $T \in \mathcal{B}(\mathcal{H})$ be normal, fix $x \in \mathcal{H}$ and let

$$
\ell_{x}(g)=\langle g(T) x, x\rangle, \quad g \in \mathscr{C}\left(\Omega_{j}^{+}, \mathbb{R}\right) .
$$

It is readily checked that $\ell_{x}$ is a real-valued bounded linear functional on $\mathscr{C}\left(\Omega_{j}^{+}, \mathbb{R}\right)$. Moreover, $\ell_{x}$ is a positive functional. Indeed, if $h$ is a continuous non-negative function on $\Omega_{j}^{+}$, then there exists a function $g$ given by $g(u, v)=\sqrt{h(u, v)}$ so that $g \in \mathscr{C}\left(\Omega_{j}^{+}, \mathbb{R}\right)$ and $g(T)=g(T)^{*}$. Thus,

$$
\begin{aligned}
\ell_{x}(h)=\langle h(T) x, x\rangle & =\langle g(T) x, g(T) x\rangle \\
& =\|g(T) x\|^{2} \geq 0 .
\end{aligned}
$$

Theorem 2.16 yields the existence of a uniquely determined positive valued measure $\mu_{x}$ (for a fixed $j \in \mathbb{S}$ ) so that

$$
\ell_{x}(g)=\int_{\Omega_{j}^{+}} g(p) d \mu_{x}(p), \quad g \in \mathscr{C}\left(\Omega_{j}^{+}, \mathbb{R}\right) .
$$

In view of (4.1), we may use the formula

$$
\begin{aligned}
4\langle T x, y\rangle & =\langle T(x+y), x+y\rangle-\langle T(x-y), x-y\rangle+e_{1}\left\langle T\left(x+y e_{1}\right), x+y e_{1}\right\rangle \\
& -e_{1}\left\langle T\left(x-y e_{1}\right), x-y e_{1}\right\rangle+e_{1}\left\langle T\left(x-y e_{2}\right), x-y e_{2}\right\rangle e_{3} \\
& -e_{1}\left\langle T\left(x+y e_{2}\right), x+y e_{2}\right\rangle e_{3}+\left\langle T\left(x+y e_{3}\right), x+y e_{3}\right\rangle e_{3} \\
& -\left\langle T\left(x-y e_{3}\right), x-y e_{3}\right\rangle e_{3},
\end{aligned}
$$

where $\left\{1, e_{1}, e_{2}, e_{3}\right\}$ denotes the standard basis of $\mathbb{H}$, to obtain a uniquely determined $\mathbb{H}$-valued measure $\mu_{x, y}$ (relative to a fixed $j \in \mathbb{S}$ ) so that

$$
\langle g(T) x, y\rangle=\int_{\Omega_{j}^{+}} g(p) d \mu_{x, y}(p), \quad g \in \mathscr{C}\left(\Omega_{j}^{+}, \mathbb{R}\right),
$$

where

$$
\begin{aligned}
4 \mu_{x, y} & =\mu_{x+y}-\mu_{x-y}+e_{1} \mu_{x+y e_{1}}-e_{1} \mu_{x-y e_{1}} \\
& +e_{1} \mu_{x-y e_{2}} e_{3}-e_{1} \mu_{x+y e_{2}} e_{3}+\mu_{x+y e_{3}} e_{3}-\mu_{x-y e_{3}} e_{3} .
\end{aligned}
$$

Definition 4.2. The Borel sets of $\sigma_{S}(T) \cap \mathbb{C}_{j}^{+}$will be denoted by $\mathfrak{B}\left(\sigma_{S}(T) \cap \mathbb{C}_{j}^{+}\right)$.

Lemma 4.3. The $\mathbb{H}$-valued measure $\mu_{x, y}$ given in (4.4) enjoys the following properties:

(i) $\mu_{x \alpha+y \beta, z}=\mu_{x, z} \alpha+\mu_{y, z} \beta, \quad \alpha, \beta \in \mathbb{H}$.

(ii) $\mu_{x, y \alpha+z \beta}=\bar{\alpha} \mu_{x, y}+\bar{\beta} \mu_{x, z}, \quad \alpha, \beta \in \mathbb{H}$.

(iii) $\left|\mu_{x, y}\left(\sigma_{S}(T) \cap \mathbb{C}_{j}^{+}\right)\right| \leq\|x\|\|y\|$.

(iv) $\bar{\mu}_{x, y}=\mu_{y, x}$,

for all $x, y, z \in \mathcal{H}$.

Proof. Properties (i)-(iii) are easily obtained from (4.3) using the uniqueness of $\mu_{x, y}$ (relative to a fixed $j \in \mathbb{S}$ ) and the properties of $\langle\cdot, \cdot\rangle$. Property (iv) follows from properties (i) and (ii). 
It follows from properties (i) and (iii) in Lemma 4.3 that $\Phi_{y}(x)=\mu_{x, y}(\sigma)$, where $y \in \mathcal{H}$ and $\sigma \in \mathfrak{B}\left(\sigma_{S}(T) \cap \mathbb{C}_{j}^{+}\right)$are fixed, is a continuous right linear functional on $\mathcal{H}$. Moreover, it follows from property (ii) in Lemma 4.3 that

$$
\Phi_{y \alpha}(x)=\bar{\alpha} \Phi_{y}(x), \quad \alpha \in \mathbb{H} .
$$

It follows from the Riesz representation theorem for quaternionic Hilbert spaces (see Theorem 6.1 in Ref. 13 and also Proposition 1.10 in Ref. 30 for a statement and proof in a more general Clifford algebra setting) that corresponding to any $x \in \mathcal{H}$, there exists a unique vector $w \in \mathcal{H}$ such that

$$
\Phi_{y}(x)=\langle x, w\rangle,
$$

i.e., $\mu_{x, y}(\sigma)=\langle x, w\rangle$. Since the left hand of (4.5) depends linearly on $x$ and anti-linearly on $y$ and the right hand side depends linearly on $x$, it follows that $\Phi_{y}(x)$ depends linearly on $x$ and anti-linearly on $y$,

$$
E(\sigma) y=w
$$

for some

$$
E(\sigma) \in \mathcal{B}(\mathcal{H})
$$

Thus,

$$
\mu_{x, y}(\sigma)=\langle x, E(\sigma) y\rangle, \quad \sigma \in \mathfrak{B}\left(\sigma_{S}(T) \cap \mathbb{C}_{j}^{+}\right),
$$

and, in view of property (iv) in Lemma 4.3,

$$
E(\sigma)=E(\sigma)^{*}, \quad \sigma \in \mathfrak{B}\left(\sigma_{S}(T) \cap \mathbb{C}_{j}^{+}\right)
$$

and hence,

$$
\mu_{x, y}(\sigma)=\langle E(\sigma) x, y\rangle, \quad \sigma \in \mathfrak{B}\left(\sigma_{S}(T) \cap \mathbb{C}_{j}^{+}\right) .
$$

Since $\mu_{x}$ is countably additive, $\mu_{x, y}$ is also countably additive. Consequently, the $\mathcal{B}(\mathcal{H})$-valued measure $E$ is also countably additive, i.e.,

$$
E\left(\bigcup_{n=0}^{\infty} \sigma_{n}\right)=\sum_{n=0}^{\infty} E\left(\sigma_{n}\right)
$$

for any sequence of pairwise disjoint sets

$$
\left\{\sigma_{n}\right\}_{n=0}^{\infty} \subseteq \mathfrak{B}\left(\sigma_{S}(T) \cap \mathbb{C}_{j}^{+}\right) .
$$

The limit in (4.8) is meant with respect to the strong operator topology.

Lemma 4.4. Fix a normal operator $T \in \mathcal{B}(\mathcal{H})$ and let $J \in \mathcal{B}(\mathcal{H})$ be as in Theorem 3.6 and $E(\sigma)$ be given by (4.7) and $\Omega_{j}^{+}=\sigma_{S}(T) \cap \mathbb{C}_{j}^{+}$for $j \in \mathbb{S}$. The following statements hold:

(i) If $g \in \mathscr{C}\left(\Omega_{j}^{+}, \mathbb{R}\right)$, then

$$
\langle g(T) x, y\rangle=\int_{\Omega_{j}^{+}} g(p) d\langle E(p) x, y\rangle, \quad x, y \in \mathcal{H} .
$$

(ii) If $\sigma_{S}(T) \cap \mathbb{R}=\emptyset$ (respectively, $\sigma_{S}(T) \cap \mathbb{R} \neq \emptyset$ ), then, for any $f=f_{0}+f_{1} j \in \mathscr{C}\left(\Omega_{j}^{+}, \mathbb{C}_{j}\right)$ (respectively, $\mathscr{C}_{0}\left(\Omega_{j}^{+}, \mathbb{C}_{j}\right)$ ), where $f_{0}$ and $f_{1}$ are real-valued,

$$
\langle f(T) x, y\rangle=\int_{\Omega_{j}^{+}} f_{0}(p) d\langle E(p) x, y\rangle+\int_{\Omega_{j}^{+}} f_{1}(p) d\langle J E(p) x, y\rangle, \quad x, y \in \mathcal{H} .
$$

(iii) $E(\sigma)$ and $J$ commute for all $\sigma \in \mathfrak{B}\left(\Omega_{j}^{+}\right)$.

Proof. Assertion (4.9) follows directly from (4.3) and (4.7). We will now prove assertion (4.10). In view of (4.9) and (3.10), 


$$
\begin{aligned}
\langle f(T) x, y\rangle & =\left\langle\left\{f_{0}(T)+f_{1}(T) J\right\} x, y\right\rangle \\
& =\left\langle f_{0}(T) x, y\right\rangle+\left\langle f_{1}(T) J x, y\right\rangle \\
& =\int_{\Omega_{j}^{+}} f_{0}(p) d\langle E(p) x, y\rangle+\int_{\Omega_{j}^{+}} f_{1}(p) d\langle E(p) J x, y\rangle, \quad x, y \in \mathcal{H} .
\end{aligned}
$$

Thus, the proof of (4.10) will be complete upon showing that

$$
d\langle E(p) J x, y\rangle=d\langle J E(p) x, y\rangle, \quad x, y \in \mathcal{H} .
$$

To see this, let $g \in \mathscr{C}\left(\Omega_{j}^{+}, \mathbb{R}\right)$ and use (4.9) and the fact that $g(T)$ and $J$ commute to obtain

$$
\int_{\Omega_{j}^{+}} g(p) d\langle E(p) J x, y\rangle=\langle g(T) J x, y\rangle=\langle J g(T) x, y\rangle=\int_{\Omega_{j}^{+}} g(p) d\langle J E(p) x, y\rangle .
$$

If we write $v=\langle E(p) J x, y\rangle$ and $\widetilde{v}=\langle J E(p) x, y\rangle$ and then

$$
v=v_{0} \mathrm{e}_{0}+v_{1} \mathrm{e}_{1}+v_{2} \mathrm{e}_{2}+v_{3} \mathrm{e}_{3}
$$

and

$$
\widetilde{v}=\widetilde{v}_{0} \mathrm{e}_{0}+\widetilde{v}_{1} \mathrm{e}_{1}+\widetilde{v}_{2} \mathrm{e}_{2}+\widetilde{v}_{3} \mathrm{e}_{3}
$$

where $v_{a}$ and $\widetilde{v}_{a}, a=0, \ldots, 3$ are real signed measures and $\left\{\mathrm{e}_{a}\right\}_{a=0}^{3}$ is the standard basis for $\mathbb{H}$, then it follows from Theorem 2.16 that

$$
v_{a}=\widetilde{v}_{a}, \quad a=0, \ldots, 3 .
$$

Therefore, items (iii) and (ii) hold.

Theorem 4.5. The $\mathcal{B}(\mathcal{H})$-valued countably additive measure E, given by (4.7), for all $\sigma, \tau \in$ $\mathfrak{B}\left(\sigma_{S}(T) \cap \mathbb{C}_{j}^{+}\right)$, enjoys the following properties:

(i) $E(\sigma)=E(\sigma)^{*}$.

(ii) $\|E(\sigma)\| \leq 1$.

(iii) $E(\emptyset)=0$ and $E\left(\sigma_{S}(T) \cap \mathbb{C}_{j}^{+}\right)=I_{\mathcal{H}}$.

(iv) $E(\sigma \cap \tau)=E(\sigma) E(\tau)$.

(v) $E(\sigma)^{2}=E(\sigma)$.

(vi) If $\sigma_{S}(T) \cap \mathbb{R}=\emptyset$ (respectively, $\sigma_{S}(T) \cap \mathbb{R} \neq \emptyset$ ), then $E(\sigma)$ commutes with $f(T)$ for all $f \in$ $\mathscr{C}\left(\sigma_{S}(T) \cap \mathbb{C}_{j}^{+}, \mathbb{C}_{j}\right)$ (respectively, $\left.f \in \mathscr{C}_{0}\left(\sigma_{S}(T) \cap \mathbb{C}_{j}^{+}, \mathbb{C}_{j}\right)\right)$.

(vii) $E(\sigma)$ and $E(\tau)$ commute.

Proof. The proof is broken into steps.

Step 1: Show (i) and (ii).

Property (i) has already been noted in (4.6). Property (ii) follows directly from property (iii) in Lemma 4.3. Indeed, if $x=y$ in property (iii) in Lemma 4.3, then

$$
\mu_{x, x}(\sigma) \leq \mu_{x, x}\left(\sigma_{S}\left(\sigma_{S}(T) \cap \mathbb{C}_{j}^{+}\right) \leq\|x\|^{2}\right.
$$

and hence

$$
\langle E(\sigma) x, x\rangle \leq\|x\|^{2} \text { for } \quad x \in \mathcal{H},
$$

i.e., $I_{\mathcal{H}}-E(\sigma)$ is a positive operator for all $\sigma \in \mathfrak{B}\left(\sigma_{S}(T) \cap \mathbb{C}_{j}^{+}\right)$. Therefore, property (ii) holds.

Step 2: Show (iii).

Since $\mu_{x, y}(\emptyset)=0$, we may use (4.3) to deduce $E(\emptyset)=0$. Similarly, putting $g(p)=1$ in (4.3) yields $g(T)=I_{\mathcal{H}}$ for all $x, y \in \mathcal{H}$ and thus

$$
\langle x, y\rangle=\int_{\sigma_{S}(T) \cap \mathbb{C}_{j}^{+}} d \mu_{x, y}=\left\langle E\left(\sigma_{S}(T) \cap \mathbb{C}_{j}^{+}\right) x, y\right\rangle,
$$

i.e., $E\left(\sigma_{S}(T) \cap \mathbb{C}_{j}^{+}\right)=I_{\mathcal{H}}$. 
Step 4: Show (iv).

It is clear that for all real-valued polynomials $\phi$ and $\psi$ on $\sigma_{S}(T) \cap \mathbb{C}_{j}$, for $j \in \mathbb{S}$ which satisfy

$$
\phi(u, v)=\phi(u,-v)
$$

and

$$
\psi(u, v)=\psi(u,-v),
$$

we have that $(\phi \psi)(T)=\phi(T) \psi(T), \phi(T)=\phi(T)^{*}$, and $\psi(T)=\psi(T)^{*}$ (see Remark 3.12). Thus,

$$
\begin{aligned}
\int_{\sigma_{S}(T) \cap \mathbb{C}_{j}^{+}} \phi(p) d \mu_{\psi(T) x, x}(p) & =\langle\phi(T) \psi(T) x, x\rangle \\
& =\langle(\phi \psi)(T) x, x\rangle \\
& =\int_{\sigma_{S}(T) \cap \mathbb{C}_{j}^{+}} \phi(p) \psi(p) d \mu_{x, x}(p) .
\end{aligned}
$$

Since $E(\sigma)=E(\sigma)^{*},(4.7)$ implies that

$$
\mu_{x, x}(\sigma) \in \mathbb{R} \text { for all } \sigma \in \mathfrak{B}\left(\sigma_{S}(T) \cap \mathbb{C}_{j}^{+}\right) .
$$

Similarly, since $\langle\psi(T) x, x\rangle$ is real, (4.7) implies that

$$
\mu_{\psi(T) x, x}(\sigma) \in \mathbb{R} \text { for all } \sigma \in \mathfrak{B}\left(\sigma_{S}(T) \cap \mathbb{C}_{j}^{+}\right) .
$$

In view of the identification given in item (i) of Lemma 4.1, the density of real-valued polynomials in the space $\mathscr{C}\left(\sigma_{S}(T) \cap \mathbb{C}_{j}^{+}, \mathbb{R}\right)$ and the Riesz representation theorem given in Theorem 2.16, (4.11) implies that

$$
d \mu_{\psi(T) x, x}(p)=\psi(p) d \mu_{x, x}(p) .
$$

But then we may use identity (4.4) and the fact that $\psi(p)$ is real-valued to obtain

$$
d \mu_{\psi(T) x, y}(p)=\psi(p) d \mu_{x, y}(p) .
$$

Thus, in view of (4.7),

$$
\langle E(\sigma) \psi(T) x, y\rangle=\int_{\sigma} \psi(p) d \mu_{x, y}(p) \text { for } \sigma \in \mathfrak{B}\left(\sigma_{S}(T) \cap \mathbb{C}_{j}^{+}\right) .
$$

Since $E(\sigma)=E(\sigma)^{*}$ for $\sigma \in \mathfrak{B}\left(\sigma_{S}(T) \cap \mathbb{C}_{j}^{+}\right)$,

$$
\int_{\sigma_{S}(T) \cap \mathbb{C}_{j}^{+}} \psi d \mu_{x, E(\sigma) y}=\langle\psi(T) x, E(\sigma) y\rangle=\langle E(\sigma) \psi(T) x, y\rangle=\int_{\sigma_{S}(T) \cap \mathbb{C}_{j}^{+}} \psi \chi_{\sigma} d \mu_{x, y},
$$

where

$$
\chi_{\sigma}(p)=\left\{\begin{array}{lll}
1 & \text { if } & p \in \sigma \\
0 & \text { if } & p \notin \sigma
\end{array} .\right.
$$

Since $\psi$ is real-valued, we also have

$$
\int_{\sigma_{S}(T) \cap \mathbb{C}_{j}^{+}} \psi d \mu_{x, E(\sigma) y}^{(m)}=\int_{\sigma_{S}(T) \cap \mathbb{C}_{j}^{+}} \psi \chi_{\sigma} d \mu_{x, y}^{(m)} \text { for } m=0, \ldots, 3,
$$

where $\mu_{x, y}^{(m)}$ and $\mu_{x, E(\sigma) y}^{(m)}$ are real-valued signed measures given by

$$
\mu_{x, y}=\sum_{m=0}^{3} \mu_{x, y}^{(m)} e_{m}
$$

and

$$
\mu_{x, E(\sigma) y}^{(m)}=\sum_{m=0}^{3} \mu_{x, E(\sigma) y}^{(m)} e_{m} .
$$

Recall that $\left\{e_{m}\right\}_{m=0}^{3}$ is the standard basis for $\mathbb{H}$. 
In view of the identification given in item (i) of Lemma 4.1, the density of real-valued polynomials in the space $\mathscr{C}\left(\sigma_{S}(T) \cap \mathbb{C}_{j}^{+}, \mathbb{R}\right)$ and the Riesz representation theorem given in Theorem 2.16, (4.12) implies that

$$
d \mu_{x, E(\sigma) y}^{(m)}=\chi_{\sigma} d \mu_{x, y}^{(m)} \text { for } \quad m=0, \ldots, 3
$$

and hence

$$
d \mu_{x, E(\sigma) y}=\chi_{\sigma} d \mu_{x, y} .
$$

Therefore,

$$
\mu_{x, E(\sigma) y}(\tau)=\int_{\sigma_{S}(T) \cap \mathbb{C}_{j}^{+} \cap \tau} \chi_{\sigma} d \mu_{x, y}=\mu_{x, y}(\sigma \cap \tau) \quad \text { for } \quad \sigma, \tau \in \mathfrak{B}\left(\sigma_{S}(T) \cap \mathbb{C}_{j}^{+}\right)
$$

and hence as

$$
\mu(\sigma)=\langle E(\sigma) x, y\rangle \text { for } \sigma \in \mathfrak{B}\left(\sigma_{S}(T) \cap \mathbb{C}_{j}^{+}\right),
$$

we obtain $E(\sigma) E(\tau)=E(\sigma \cap \tau)$ for $\sigma, \tau \in \mathfrak{B}\left(\sigma_{S}(T) \cap \mathbb{C}_{j}^{+}\right)$.

Step 5: Show (v).

Property (v) can be obtained from Property (iv) when $\sigma=\tau$.

Step 6: Show (vi).

Let $A, B$, and $J$ be as in Theorem 3.6. We have already observed in item (iii) of Lemma 4.4 that $E(\sigma)$ and $J$ commute. One can show in a similar fashion that $A$ and $E(\sigma)$ commute and $B$ and $E(\sigma)$ commute. Thus, in view of the construction of $f(T)$ given in Remark 3.12, $f(T)$ and $E(\sigma)$ commute.

Step 7: Show (vii).

Property (vii) follows from Property (iv) interchanging $\tau$ and $\sigma$.

Definition 4.6. A $\mathcal{B}(\mathcal{H})$-valued measure $E$ on $\sigma_{S}(T) \cap \mathbb{C}_{j}^{+}$will be called a spectral measure if $E$ has Properties (i)-(vi) in Theorem 4.5. Note that $E(\sigma)$ is a positive operator for all $\sigma \in \mathfrak{B}\left(\sigma_{S}(T) \cap \mathbb{C}_{j}^{+}\right)$.

We are now ready to state and prove the main result of the section.

Theorem 4.7. Suppose $T \in \mathcal{B}(\mathcal{H})$ is normal, let $J \in \mathcal{B}(\mathcal{H})$ be as in the decomposition of Theorem 3.6 and fix $j \in \mathbb{S}$. Let $\Omega_{j}^{+}=\sigma_{S}(T) \cap \mathbb{C}_{j}^{+}$and $\Pi_{ \pm}^{j}$ denote the orthogonal projection onto $\mathcal{H}_{ \pm}^{j}$ given in (3.3), respectively. If $\sigma_{S}(T) \cap \mathbb{R}=\emptyset$ (respectively, $\sigma_{S}(T) \cap \mathbb{R} \neq \emptyset$ ), then there exists a unique spectral measure $E_{j}$ on $\Omega_{j}^{+}$so that

$$
\langle f(T) x, y\rangle=\int_{\Omega_{j}^{+}} f_{0}(p) d\left\langle E_{j}(p) x, y\right\rangle+\int_{\Omega_{j}^{+}} f_{1}(p) d\left\langle J E_{j}(p) x, y\right\rangle, \quad x, y \in \mathcal{H},
$$

or, equivalently,

$$
\langle f(T) x, y\rangle=\int_{\Omega_{j}^{+}} f(p) d\left\langle\Pi_{+}^{j} E_{j}(p) x, y\right\rangle+\int_{\Omega_{j}^{+}} \overline{f(p)} d\left\langle\Pi_{-}^{j} E_{j}(p) x, y\right\rangle, \quad x, y \in \mathcal{H},
$$

for $f=f_{0}+f_{1 j} \in \mathscr{C}\left(\Omega_{j}^{+}, \mathbb{C}_{j}\right)$ (respectively, $\mathscr{C}_{0}\left(\Omega_{j}^{+}, \mathbb{C}_{j}\right)$ ), where $f_{0}$ and $f_{1}$ are real-valued. Moreover, upon identifying the complex plane $\mathbb{C}_{k}$ with $\mathbb{C}_{j}$ in the natural way by the mapping $\varphi_{k j}$, we have $E_{j}\left(\varphi_{k j}(\sigma)\right)=E_{k}(\sigma), \sigma \in \mathfrak{B}\left(\Omega_{k}^{+}\right)$for all $j, k \in \mathbb{S}$.

Remark 4.8. In point of fact, (4.13) holds for a much larger class of functions than $\mathscr{C}\left(\sigma_{S}(T) \cap\right.$ $\left.\mathbb{C}_{j}^{+}, \mathbb{C}_{j}\right)$ or $\mathscr{C}_{0}\left(\sigma_{S}(T) \cap \mathbb{C}_{j}^{+}, \mathbb{C}_{j}\right)$ regardless of whether or not $\sigma_{S}(T) \cap \mathbb{R} \neq \emptyset$ (see Section V).

Proof of Theorem 4.7. Formula (4.13) was established in item (ii) of Lemma 4.4. Formula (4.14) follows from (4.13). Indeed, if we write $y=y_{+}+y_{-} \in \mathcal{H}$ with respect to the decomposition 
$\mathcal{H}=\mathcal{H}_{+}^{j} \oplus \mathcal{H}_{-}^{j}($ see $(3.3))$ and use (3.2), then

$$
\begin{aligned}
\langle f(T) x, y\rangle= & \int_{\Omega_{j}^{+}} f_{0}(p) d\left\langle E_{j}(p) x, y\right\rangle+\int_{\Omega_{j}^{+}} f_{1}(p) d\left\langle E_{j}(p) x,-J\left(y_{+}+y_{-}\right)\right\rangle \\
= & \int_{\Omega_{j}^{+}} f_{0}(p) d\left\langle E_{j}(p) x, y\right\rangle-\int_{\Omega_{j}^{+}} f_{1}(p) d\left\langle E_{j}(p) x, y_{+} j\right\rangle \\
& +\int_{\Omega_{j}^{+}} f_{1}(p) d\left\langle E_{j}(p) x, y_{-} j\right\rangle \\
= & \int_{\Omega_{j}^{+}} f(p) d\left\langle E_{j}(p) x, y_{+}\right\rangle+\int_{\Omega_{j}^{+}} \overline{f(p)} d\left\langle E_{j}(p) x, y-\right\rangle \\
= & \int_{\Omega_{j}^{+}} f(p) d\left\langle\Pi_{+}^{j} E_{j}(p) x, y\right\rangle+\int_{\Omega_{j}^{+}} \overline{f(p)} d\left\langle\Pi_{-}^{j} E_{j}(p) x, y\right\rangle .
\end{aligned}
$$

The fact that there is only one spectral measure $E_{j}$ on $\sigma_{S}(T) \cap \mathbb{C}_{j}^{+}$such that (4.13) holds follows directly from the uniqueness of the measure $\mu_{x, y}(\sigma)=\langle E(\sigma) x, y\rangle$ on $\Omega_{j}^{+}$(see (4.4)). The claimed invariance $E_{j}\left(\varphi_{j k}(\sigma)\right)=E_{k}(\sigma)$ relative to $j, k \in \mathbb{S}$ drops out easily from the aforementioned uniqueness of $E_{j}$ and Theorem 2.14.

Corollary 4.9. In the setting of Theorem 4.7, the following statements hold:

(i) If $T \in \mathcal{B}(\mathcal{H})$ is a positive operator, then there exists a unique positive operator $T^{1 / 2}:=W \in$ $\mathcal{B}(\mathcal{H})$ so that $W^{2}=T$.

(ii) $\quad T \in \mathcal{B}(\mathcal{H})$ is self-adjoint if and only if

$$
\langle T x, y\rangle=\int_{[-\|T\|,\|T\|]} t d\left\langle E_{j}(t) x, y\right\rangle, \quad x, y \in \mathcal{H} .
$$

(iii) $\quad T \in \mathcal{B}(\mathcal{H})$ is anti self-adjoint if and only if

$$
\langle T x, y\rangle=\int_{[0,\|T\|]} t d\left\langle J E_{j}(t) x, y\right\rangle, \quad x, y \in \mathcal{H} .
$$

(iv) $\quad T \in \mathcal{B}(\mathcal{H})$ is unitary if and only if

$$
\langle T x, y\rangle=\int_{[0, \pi]} \cos (t) d\left\langle E_{j}(t) x, y\right\rangle+\int_{[0, \pi]} \sin (t) d\left\langle J E_{j}(t) x, y\right\rangle .
$$

Proof. As we have observed in item (i) of Theorem 2.15, if $T \in \mathcal{B}(\mathcal{H})$ is a positive operator, then $\sigma_{S}(T) \subseteq[0,\|T\|]$. Thus, using Theorem 4.7 we have the existence of a uniquely determined spectral measure $E$ so that

$$
\langle T x, y\rangle=\int_{[0,\|T\|]} t d\left\langle E_{j}(t) x, y\right\rangle .
$$

Let $g(t)=t^{1 / 2}$ for $t \in \mathbb{R}$. Since $g \in \mathscr{C}\left(\sigma_{S}(T), \mathbb{R}\right)$, it follows from Theorem 4.7 that

$$
\langle W x, y\rangle:=\langle g(T) x, y\rangle=\int_{[0,\|T\|]} t^{1 / 2} d\left\langle E_{j}(t) x, y\right\rangle
$$

satisfies $W^{2}=T$. Thus, we have established the existence of a positive operator $W \in \mathcal{B}(\mathcal{H})$ so that $W^{2}=T$. The proof that $W$ is unique follows from the uniqueness of the spectral measure $E$, just as in the case that $\mathcal{H}$ is a complex Hilbert space.

The proofs of (ii)-(iv) follow readily from Theorem 4.7 and (2.8).

\section{SPECTRAL INTEGRALS}

The goal of this section is to extend the integral representation in Theorem 4.7 to a more general class of functions. This will be useful when proving a spectral theorem for unbounded operators in 
Section VI. To this end we will adapt part of Chapter 4 of the book ${ }^{33}$ to the quaternionic setting. Most of the proofs of the properties of spectral integrals are easily adapted from the classical case presented in Ref. 33, i.e., when $\mathcal{H}$ is a complex Hilbert space. However, some facts require additional arguments which we will highlight.

Throughout this section, we will fix $j \in \mathbb{S}$, a spectral measure $E$ (see Definition 4.6) on a set $\Omega \subseteq \mathbb{C}_{j}$ and an anti self-adjoint and unitary operator $J \in \mathcal{B}(\mathcal{H})$ which commutes with $E$.

Definition 5.1. Let $\mathscr{B}\left(E, \Omega, \mathbb{C}_{j}\right)$ denote the set of all bounded $\Omega$-measurable $\mathbb{C}_{j}$-valued functions $f$ on $\Omega$ with the norm

$$
\|f\|_{\infty}=\sup _{p \in \Omega}|f(p)| .
$$

Let $\mathscr{B}_{s}\left(E, \Omega, \mathbb{C}_{j}\right)$ denote the subset of simple functions in $\mathscr{B}\left(E, \Omega, \mathbb{C}_{j}\right)$, i.e., all $\mathbb{C}_{j}$-valued functions of the form $f(p)=\sum_{m=1}^{n} c_{m} \chi_{\sigma_{m}}(p)$, where $\sigma_{1}, \ldots, \sigma_{n}$ are pairwise disjoint sets in $\mathfrak{B}(\Omega), c_{1}, \ldots, c_{n} \in \mathbb{C}_{j}$ and

$$
\chi_{\sigma}(p)=\left\{\begin{array}{lll}
1 & \text { if } & p \in \sigma \\
0 & \text { if } & p \notin \sigma
\end{array} .\right.
$$

If $f \in \mathscr{B}_{s}\left(E, \Omega, \mathbb{C}_{j}\right)$, then we may define

$$
\mathbb{I}(f)=\int_{\Omega} f(p) d E(p):=\sum_{m=1}^{n}\left\{\operatorname{Re}\left(c_{m}\right) I_{\mathcal{H}}+\operatorname{Im}\left(c_{m}\right) J\right\} E\left(\sigma_{m}\right),
$$

and

$$
\mathbb{I}(\bar{f})=\int_{\Omega} \overline{f(p)} d E(p):=\sum_{m=1}^{n}\left\{\operatorname{Re}\left(c_{m}\right) I_{\mathcal{H}}-\operatorname{Im}\left(c_{m}\right) J\right\} E\left(\sigma_{m}\right)
$$

Lemma 5.2. If $f \in \mathscr{B}_{s}\left(E, \Omega, \mathbb{C}_{j}\right)$, then

$$
\|\mathbb{I}(f)\| \leq\|f\|_{\infty} .
$$

Proof. If $f=\sum_{m=1}^{n} c_{m} \chi_{\sigma_{m}}$, where $c_{1}, \ldots, c_{n} \in \mathbb{C}_{j}$ and $\sigma_{1}, \ldots, \sigma_{n}$ are disjoint sets in $\mathfrak{B}(\Omega)$, then, using properties (ii)-(iv) in Theorem 4.5 and the fact that $\|J\|=1$,

$$
\begin{aligned}
\|\mathbb{I}(f) x\|^{2} & =\left\|\sum_{m=1}^{n}\left\{\operatorname{Re}\left(c_{m}\right)+\operatorname{Im}\left(c_{m}\right) J\right\} E\left(\sigma_{m}\right) x\right\|^{2} \\
& =\sum_{m=1}^{n}\left\|\left\{\operatorname{Re}\left(c_{m}\right)+\operatorname{Im}\left(c_{m}\right) J\right\} E\left(\sigma_{m}\right) x\right\|^{2} \\
& \leq \sum_{m=1}^{n}\left|c_{m}\right|^{2}\left\|E\left(\sigma_{m}\right) x\right\|^{2} \\
& \leq\|f\|_{\infty}^{2}\|x\|^{2} .
\end{aligned}
$$

Thus, (5.2) holds.

Fix $f \in \mathscr{B}\left(E, \Omega, \mathbb{C}_{j}\right)$. Since $\mathscr{B}_{s}\left(E, \Omega, \mathbb{C}_{j}\right)$ is a dense subset of $\mathscr{B}\left(E, \Omega, \mathbb{C}_{j}\right)$, there exists a sequence of functions $\left\{f_{n}\right\}_{n=0}^{\infty}$ belonging to $\mathscr{B}_{s}\left(E, \Omega, \mathbb{C}_{j}\right)$ so that

$$
\lim _{n \uparrow \infty}\left\|f_{n}-f\right\|_{\infty}=0 .
$$

In view of (5.2), $\left\{\mathbb{I}\left(f_{n}\right) x\right\}_{n=0}^{\infty}$ is a Cauchy sequence in $\mathcal{H}$. Let $\mathbb{I}(f)$ be given by

$$
\mathbb{I}(f) x=\lim _{n \uparrow \infty} \mathbb{I}\left(f_{n}\right) x, \quad x \in \mathcal{H} .
$$

Note that $f$ does not depend on the choice of the sequence $\left\{f_{n}\right\}_{n=0}^{\infty}$ and, consequently, neither does $\mathbb{I}(f)$. 
Lemma 5.3. If $f=f_{0}+f_{1} j$ and $g=g_{0}+g_{1} j$ belong to $\mathscr{B}\left(E, \Omega, \mathbb{C}_{j}\right)$, where $f_{0}, f_{1}, g_{0}$, and $g_{1}$ are real-valued, $\alpha, \beta \in \mathbb{C}_{j}$ and $x, y \in \mathcal{H}$, then

(i) $\quad \mathbb{I}(f)^{*}=\mathbb{I}(\bar{f}), \quad \mathbb{I}(\alpha f+\beta g)=\alpha \mathbb{I}(f)+\beta \mathbb{I}(g)$.

(ii) $\langle\mathbb{I}(f) x, y\rangle=\int_{\Omega} f_{0}(p) d\langle E(p) x, y\rangle+\int_{\Omega} f_{1}(p) d\langle J E(p) x, y\rangle$.

(iii) $\mathbb{I}(f g)=\mathbb{I}(f) \mathbb{I}(g)$.

(iv) $\quad\|\mathbb{I}(f) x\|^{2}=\int_{\Omega}|f(p)|^{2} d\langle E(p) x, x\rangle$.

(v) $\quad\|\mathbb{I}(f)\| \leq\|f\|_{\infty}$.

Proof. In view of the density of $\mathscr{B}_{s}\left(E, \Omega, \mathbb{C}_{j}\right)$ in $\mathscr{B}\left(E, \Omega, \mathbb{C}_{j}\right)$ and (5.2), it suffices to check (i)-(v) when $f, g \in \mathscr{B}_{s}\left(E, \Omega, \mathbb{C}_{j}\right)$. The assumption that $E(\sigma)$ and $J$ commute for all $\sigma \in \mathfrak{B}(\Omega)$ will be a useful tool for checking (i)-(v) and will be used without further mention. If $f=\sum_{m=1}^{n} c_{m} \chi_{\sigma_{m}}$ and $g=\sum_{m=1}^{n} d_{m} \chi_{\tau_{m}}$ belong to $\mathscr{B}_{s}\left(E, \Omega, \mathbb{C}_{j}\right)$, then

$$
\begin{aligned}
\left\langle\mathbb{I}(f)^{*} y, x\right\rangle & =\sum_{m=1}^{n}\left\langle y,\left\{\operatorname{Re}\left(c_{m}\right) I_{\mathcal{H}}+\operatorname{Im}\left(c_{m}\right) J\right\} E\left(\sigma_{m}\right) x\right\rangle \\
& =\sum_{m=1}^{n}\left\langle\left\{\operatorname{Re}\left(c_{m}\right) I_{\mathcal{H}}-\operatorname{Im}\left(c_{m}\right) J\right\} E\left(\sigma_{m}\right) y, x\right\rangle \\
& =\langle\mathbb{I}(\bar{f}) y, x\rangle, \quad x, y \in \mathcal{H} .
\end{aligned}
$$

Thus, the first assertion in (i) holds. The second assertion in (i) is easily checked.

We will now check that (ii) holds. Since

$$
\begin{aligned}
\langle\mathbb{I}(f) x, y\rangle & =\sum_{m=1}^{n}\left\{\operatorname{Re}\left(c_{m}\right)\left\langle E\left(\sigma_{m}\right) x, y\right\rangle+\operatorname{Im}\left(c_{m}\right)\left\langle J E\left(\sigma_{m}\right) x, y\right\rangle\right\} \\
& =\int_{\Omega} f_{0}(p) d\langle E(p) x, y\rangle+\int_{\Omega} f_{1}(p) d\langle J E(p) x, y\rangle,
\end{aligned}
$$

where $f_{0}$ and $f_{1}$ are real-valued functions which satisfy $f=f_{0}+f_{1} j$, (ii) holds.

We will now check that (iii) holds. Since

$$
\begin{aligned}
\mathbb{I}(f g) & =\sum_{\ell, m=1}^{n}\left\{\operatorname{Re}\left(c_{\ell}\right) \operatorname{Re}\left(d_{m}\right)-\operatorname{Im}\left(c_{\ell}\right) \operatorname{Im}\left(d_{m}\right)\right\} E\left(\sigma_{\ell} \cap \tau_{m}\right) \\
& +\sum_{\ell, m=1}^{n}\left\{\operatorname{Re}\left(c_{\ell}\right) \operatorname{Im}\left(d_{m}\right)+\operatorname{Im}\left(c_{\ell}\right) \operatorname{Re}\left(d_{m}\right)\right\} J E\left(\sigma_{\ell} \cap \tau_{m}\right) \\
& =\sum_{\ell, m=1}^{n}\left\{\operatorname{Re}\left(c_{\ell}\right) I_{\mathcal{H}}+\operatorname{Im}\left(c_{\ell}\right) J\right\}\left\{\operatorname{Re}\left(d_{m}\right) I_{\mathcal{H}}+\operatorname{Im}\left(d_{m}\right) J\right\} E\left(\sigma_{m}\right) E\left(\tau_{n}\right) \\
& =\left\{\sum_{\ell=1}^{n}\left\{\operatorname{Re}\left(c_{\ell}\right) I_{\mathcal{H}}+\operatorname{Im}\left(c_{\ell}\right) J\right\} E\left(\sigma_{\ell}\right)\right\}\left\{\sum_{m=1}^{n}\left\{\operatorname{Re}\left(d_{m}\right) I_{\mathcal{H}}+\operatorname{Im}\left(d_{m}\right) J\right\} E\left(\tau_{m}\right)\right\} \\
& =\mathbb{I}(f) \mathbb{I}(g),
\end{aligned}
$$

(iii) holds.

Assertion (iv) is a direct consequence of (i)-(iii). Indeed,

$$
\begin{aligned}
\|\mathbb{I}(f) x\|^{2} & =\langle\mathbb{I}(f) x, \mathbb{I}(f) x\rangle \\
& =\left\langle\mathbb{I}\left(|f|^{2}\right) x, x\right\rangle \\
& =\int_{\Omega_{j}^{+}}|f(p)|^{2} d\langle E(p) x, x\rangle, \quad x \in \mathcal{H} .
\end{aligned}
$$

Finally, assertion (v) is a direct consequence of assertion (iv).

Remark 5.4. If $T \in \mathcal{B}(\mathcal{H})$ is normal and $E_{j}$ is the spectral measure on $\sigma_{S}(T) \cap \mathbb{C}_{j}^{+}$for $j \in \mathbb{S}$ appearing in Theorem 4.7, then item (ii) of Lemma 5.3 ensures that

$$
f(T)=\mathbb{I}(f), \quad f \in \mathscr{C}\left(\sigma_{S}(T) \cap \mathbb{C}_{j}^{+}, \mathbb{C}_{j}\right) .
$$


We will now extend the functional calculus for functions in $\mathscr{B}\left(E, \Omega_{j}^{+}, \mathbb{C}_{j}\right)$ to a more general class of functions which will be useful when proving the spectral theorem for unbounded normal operators.

Definition 5.5. Let $\mathscr{B}_{\infty}\left(E, \Omega, \mathbb{C}_{j}\right)$ denote the space of all $\mathfrak{B}(\Omega)$-measurable functions $f: \Omega \rightarrow$ $\mathbb{C}_{j} \cup\{\infty\}$ which satisfy

$$
E(\{p \in \Omega: f(p)=\infty\})=0,
$$

where $E$ is a spectral measure.

Definition 5.6. A sequence of sets $\left\{\sigma_{n}\right\}_{n=0}^{\infty}$, where $\sigma_{n} \in \mathfrak{B}(\Omega)$ for $n=0,1, \ldots$ is called a bounding sequence for a subset of functions $\mathfrak{F} \subseteq \mathscr{B}_{\infty}\left(E, \Omega, \mathbb{C}_{j}\right)$ if

(i) $f \in \mathfrak{F}$ is bounded on $\sigma_{n}$ for $n=0,1, \ldots$,

(ii) $\sigma_{n} \subseteq \sigma_{n+1}$ for $n=0,1, \ldots$,

(iii) $E\left(\cup_{n=0}^{\infty} \sigma_{n}\right)=I_{\mathcal{H}}$,

where $E$ is a spectral measure.

Remark 5.7. If $\left\{\sigma_{n}\right\}_{n=0}^{\infty}$ is a bounding sequence, then the following assertions follow from the definition of a spectral measure (see Definition 4.6):

(i) $E\left(\sigma_{n}\right) \leq E\left(\sigma_{n+1}\right)$.

(ii) $E\left(\sigma_{n}\right) x \rightarrow x$ as $n \uparrow \infty, x \in \mathcal{H}$.

(iii) The set $\bigcup_{n=0}^{\infty} E\left(\sigma_{n}\right) \mathcal{H}$ is dense in $\mathcal{H}$.

We will now give meaning to $\mathbb{I}(f)$ for $f \in \mathscr{B}_{\infty}\left(E, \Omega, \mathbb{C}_{j}\right)$.

Definition 5.8. Fix $f \in \mathscr{B}_{\infty}\left(E, \Omega, \mathbb{C}_{j}\right)$ and let $\left\{\sigma_{n}\right\}_{n=0}^{\infty}$ be a bounding sequence for $f$. We let

$$
\mathbb{I}(f) x=\lim _{n \uparrow \infty} \mathbb{I}\left(\chi_{\sigma_{n}} f\right) x
$$

with domain

$$
\mathcal{D}(\mathbb{I}(f))=\left\{x \in \mathcal{H}: \int_{\Omega}|f(p)|^{2} d\langle E(p) x, x\rangle<\infty\right\} .
$$

Given a quaternionic measure $\mu$ on $\Omega \subseteq \mathbb{C}_{j}$, we will let $L_{2}(\Omega, \mu)$ consist of all measurable functions such that

$$
\|f\|_{L_{2}(\Omega, \mu)}=\left(\int_{\Omega}|f(p)|^{2} d|\mu|(p)\right)^{1 / 2}<\infty,
$$

where $|\mu|$ denotes the total variation of $\mu$ defined by

$$
|\mu|(\sigma)=\sup _{\sigma=\sqcup_{m=1}^{\infty} \sigma_{m}}\left|\mu\left(\sigma_{m}\right)\right|, \quad \sigma \in \mathfrak{B}(\Omega) .
$$

Here $\sqcup$ denotes a disjoint union.

Lemma 5.9. If $E$ is a spectral measure on $\Omega \subseteq \mathbb{C}_{j}$ and $\mu_{x, y}(\sigma)=\langle E(\sigma) x, y\rangle, x, y \in \mathcal{H}$, then

(i) $\left|\mu_{x, y}(\sigma)\right| \leq \mu_{x}(\sigma)^{1 / 2} \mu_{y}(\sigma)^{1 / 2}$ for $\sigma \in \mathfrak{B}(\Omega)$.

(ii) If $f \in L_{2}\left(\Omega, \mu_{x}\right)$ and $g \in L_{2}\left(\Omega, \mu_{y}\right)$, then

$$
\left.\left|\int_{\Omega} \operatorname{Re}\{(f g)(p)\} d \mu_{x, y}(p)+\int_{\Omega} \operatorname{Im}\{(f g)(p)\} d \mu_{x,-J}(p)\right| \leq 2\|f\|_{L_{2}\left(\Omega, \mu_{x}\right)}\|g\|_{L_{2}\left(\Omega, \mu_{J} y\right.}\right) .
$$

Proof. The proof of Lemma 4.8(i) in Ref. 33 can easily be adapted to obtain item (i) in our present setting. Since

$$
\begin{aligned}
\left|\int_{\Omega} \operatorname{Re}\{(f \bar{g})(p)\} d \mu_{x, y}(p)\right| & \leq \int_{\Omega}|\operatorname{Re}\{(f \bar{g})(p)\}| d\left|\mu_{x, y}\right|(p) \\
& \leq \int_{\Omega}|(f \bar{g})(p)| d\left|\mu_{x, y}\right|(p)
\end{aligned}
$$


one may proceed as in the proof of Lemma 4.8(ii) in Ref. 33 to obtain

$$
\int_{\Omega}|(f \bar{g})(p)| d\left|\mu_{x, y}\right|(p) \leq\|f\|_{L_{2}\left(\Omega, \mu_{x, x}\right)}\|g\|_{L_{2}\left(\Omega, \mu_{y, y}\right)}
$$

and hence

$$
\left|\int_{\Omega} \operatorname{Re}\{(f \bar{g})(p)\} d \mu_{x, y}(p)\right| \leq\|f\|_{L_{2}\left(\Omega, \mu_{x, x}\right)}\|g\|_{L_{2}\left(\Omega, \mu_{y, y}\right)} .
$$

Similarly, one can show that

$$
\left.\left|\int_{\Omega} \operatorname{Im}\{(f \bar{g})(p)\} d \mu_{x, y}(p)\right| \leq\|f\|_{L_{2}\left(\Omega, \mu_{x, x}\right)}\|g\|_{L_{2}\left(\Omega, \mu_{-J},-J y\right.}\right) .
$$

But since

$$
\mu_{-J y,-J y}(\sigma)=\langle E(\sigma)(-J y),-J y\rangle=\langle E(\sigma) y, y\rangle=\mu_{y, y}(\sigma),
$$

we have the advertised upper bound.

Lemma 5.10. If $f \in \mathscr{B}_{\infty}\left(E, \Omega, \mathbb{C}_{j}\right)$ and $\left\{\sigma_{n}\right\}_{n=0}^{\infty}$ is a bounding sequence for $f$, then

(i) A vector $x$ belongs to $\mathcal{D}(\mathbb{I}(f))$ if and only if the sequence $\left\{\mathbb{I}\left(\chi_{\sigma_{n}} f\right) x\right\}_{n=0}^{\infty}$ converges in $\mathcal{H}$, or, equivalently,

$$
\sup _{n=0,1, \ldots}\left\|\mathbb{I}\left(f \chi_{\sigma_{n}}\right) x\right\|<\infty .
$$

(ii) $\mathbb{I}(f)$ does not depend on the choice of bounding sequence for $f$.

(iii) The set $\cup_{n=0}^{\infty} E\left(\sigma_{n}\right) \mathcal{H}$ is a dense subset of $\mathcal{D}(\mathbb{I}(f))$ with respect to the norm $\|x\|_{\mathbb{I}(f)}=\|x\|+$ $\|\mathbb{I}(f) x\|, x \in \mathcal{D}(\mathbb{I}(f))$. Moreover,

$$
E\left(\sigma_{n}\right) \mathbb{I}(f) \subseteq \mathbb{I}(f) E\left(\sigma_{n}\right)=\mathbb{I}\left(f \chi_{\sigma_{n}}\right), \quad n=0,1, \ldots
$$

Proof. We have already observed in Definition 4.6 that $E(\sigma)$ is a positive operator on $\Omega$ for every $\sigma \in \mathfrak{B}(\Omega)$. Thus, $\mu_{x}$ is a positive measure on $\Omega$, where $\mu_{x}(\sigma)=\langle E(\sigma) x, x\rangle$. Consequently, the proof of items (i)-(iii) can be completed in much the same way as in items (i)-(iii) of Theorem 4.13 in Ref. 33.

In the following theorem, $\bar{W}$ shall denote the closure of an operator $W \in \mathcal{L}(\mathcal{H})$, while $\bar{f}$ denotes the usual conjugation of the function $f$.

Theorem 5.11. If $f, g \in \mathscr{B}_{\infty}\left(E, \Omega, \mathbb{C}_{j}\right)$ and $\alpha, \beta \in \mathbb{C}_{j}$, then

(i) $\mathbb{I}(\bar{f})=\mathbb{I}(f)^{*}$.

(ii) $\mathbb{I}(\alpha f+\beta g)=\overline{\alpha \mathbb{I}(f)+\beta \mathbb{I}(g)}$.

(iii) $\mathbb{I}(f g)=\overline{\mathbb{I}(f) \mathbb{I}(g)}$.

(iv) $\mathbb{I}(f)$ is a closed normal operator on $\mathcal{H}$ and

$$
\mathbb{I}(f)^{*} \mathbb{I}(f)=\mathbb{I}(f \bar{f})=\mathbb{I}(\bar{f} f) .
$$

(v) $\mathcal{D}(\mathbb{I}(f) \mathbb{I}(g))=\mathcal{D}(\mathbb{I}(g)) \cap \mathcal{D}(\mathbb{I}(f g))$.

(vi) If $x \in \mathcal{D}(\mathbb{I}(f))$ and $y \in \mathcal{D}(\mathbb{I}(g))$, then

$$
\langle\mathbb{I}(f) x, \mathbb{I}(g) y\rangle=\int_{\Omega} \operatorname{Re}(f(p) \overline{g(p)}) d\langle E(p) x, y\rangle+\int_{\Omega} \operatorname{Im}(f(p) \overline{g(p)}) d\langle J E(p) x, y\rangle .
$$

(vii) If $x \in \mathcal{D}(\mathbb{I}(f))$, then

$$
\|\mathbb{I}(f) x\|^{2}=\int_{\Omega}|f(p)|^{2} d\langle E(p) x, x\rangle .
$$

Proof. The proof of items (i)-(iv) when $\mathcal{H}$ is a complex Hilbert space (see items (i)-(v) of Theorem 4.16 in Ref. 33) can easily be adapted to the case when $\mathcal{H}$ is a quaternionic Hilbert space. Item 
(vii) follows directly from item (vi) when $g=f$ and $x=y$. What remains is to show (vi). To this end, we will adapt the argument for the proof of Proposition 4.15 in Ref. 33.

In view of items (i) and (ii) of Lemma 5.10,

$$
\begin{aligned}
& \int_{\Omega} \operatorname{Re}\left\{\left(f \bar{g} \chi_{m}\right)(p)\right\} d\langle E(p) x, y\rangle+\int_{\Omega} \operatorname{Im}\left\{\left(f \bar{g} \chi_{m}\right)(p)\right\} d\langle J E(p) x, y\rangle \\
= & \left\langle\mathbb{I}\left(f \bar{g} \chi_{\sigma_{m}}\right) x, y\right\rangle \\
= & \left\langle\mathbb{I}\left(f \chi_{\sigma_{m}}\right) x, \mathbb{I}\left(g \chi_{\sigma_{m}}\right) y\right\rangle .
\end{aligned}
$$

Since $x \in \mathcal{D}(\mathbb{I}(f))$ and $y \in \mathcal{D}(\mathbb{I}(g)), f \in L_{2}\left(\Omega, \mu_{x, x}\right)$ and $g \in L_{2}\left(\Omega, \mu_{y, y}\right)$, where $\mu_{x, y}(\sigma)=$ $\langle E(\sigma) x, y\rangle, \sigma \in \mathfrak{B}(\Omega)$. Therefore, we may use Lemma 5.9 to get that the integrals given in

$$
\kappa_{m}:=\int_{\Omega} \operatorname{Re}\left\{\left(f \bar{g} \chi_{m}\right)(p)\right\} d\langle E(p) x, y\rangle+\int_{\Omega} \operatorname{Im}\left\{\left(f \bar{g}_{\chi_{m}}\right)(p)\right\} d\langle J E(p) x, y\rangle
$$

exist and hence

$$
\left|\int_{\Omega} \operatorname{Re}\left\{\left(f \bar{g} \chi_{\sigma_{m}}\right)(p)\right\} d\langle E(p) x, y\rangle+\int_{\Omega} \operatorname{Im}\left\{\left(f \bar{g} \chi_{\sigma_{m}}\right)(p)\right\} d\langle J E(p) x, y\rangle-\kappa_{m}\right| \rightarrow 0
$$

as $m \uparrow \infty$. But then the formula advertised in (vi) follows from letting $m \uparrow \infty$ in (5.7).

Theorem 5.12. If $f \in \mathscr{B}_{\infty}\left(E, \Omega, \mathbb{C}_{j}\right)$, then $\mathbb{I}(f)$ is invertible if and only if $f$ does not vanish $E$-a.e. on $\Omega$. In this case,

$$
\mathbb{I}(f)^{-1}=\mathbb{I}(1 / f),
$$

where we use the convention that $1 / 0=\infty$ and $1 / \infty=0$.

Proof. The proof when $\mathcal{H}$ is a complex Hilbert space (see Proposition 4.19 in Ref. 33) can easily be adapted to the case when $\mathcal{H}$ is a quaternionic Hilbert space.

Lemma 5.13. If $g: \Omega \rightarrow \widetilde{\Omega} \subseteq \mathbb{C}_{j}, h \in \mathscr{B}_{\infty}\left(\widetilde{E}, \widetilde{\Omega}, \mathbb{C}_{j}\right)$, and $\widetilde{E}$ is the spectral measure on $\tilde{\Omega}$ given by

$$
\widetilde{E}(\widetilde{\sigma})=E\left(g^{-1}(\widetilde{\sigma})\right), \quad \widetilde{\sigma} \in \mathfrak{B}(\widetilde{\Omega}),
$$

then $\widetilde{E}(\widetilde{\sigma})$ and $J$ commute for all $\widetilde{\sigma} \in \mathfrak{B}(\widetilde{\Omega}), h \circ g \in \mathscr{B}_{\infty}\left(E, \Omega, \mathbb{C}_{j}\right)$, and

$$
\mathbb{I}_{\widetilde{E}}(h)=\mathbb{I}_{E}(h \circ g) \text {, }
$$

i.e.,

$$
\begin{aligned}
& \int_{\widetilde{\Omega}} \operatorname{Re}\{h(\tilde{p})\} d\langle\widetilde{E}(\tilde{p}) x, y\rangle+\int_{\widetilde{\Omega}} \operatorname{Im}\{h(\tilde{p})\} d\langle J \widetilde{E}(\tilde{p}) x, y\rangle \\
& =\int_{\Omega} \operatorname{Re}\{h(g(p))\} d\langle E(p) x, y\rangle+\int_{\Omega} \operatorname{Im}\{h(g(p))\} d\langle J E(p) x, y\rangle,
\end{aligned}
$$

for all $x \in \mathcal{D}\left(\mathbb{I}_{\widetilde{E}}(h)\right)=\mathcal{D}\left(\mathbb{I}_{E}(h \circ g)\right)$, where $\mathbb{I}_{\widetilde{E}}$ and $\mathbb{I}_{E}$ denote the spectral integrals with respect to $\widetilde{E}$ and $E$, respectively.

Proof. One can reason as in the proof of Proposition 4.24 in Ref. 33 (with the caveat that polarization formula (4.2) must be used) to get

$$
\begin{aligned}
\mathcal{D}\left(\mathbb{I}_{\widetilde{E}}(h)\right) & =\mathcal{D}\left(\mathbb{I}_{E}(h \circ g)\right), \\
\int_{\widetilde{\Omega}} \operatorname{Re}(h(\tilde{p})) d\langle\widetilde{E}(\tilde{p}) x, y\rangle & =\int_{\Omega} \operatorname{Re}(h(g(p)) d\langle E(p) x, y\rangle,
\end{aligned}
$$

and

$$
\int_{\widetilde{\Omega}} \operatorname{Im}(h(\tilde{p})) d\langle\widetilde{E}(\tilde{p}) x,-J y\rangle=\int_{\Omega} \operatorname{Im}(h(g(p)) d\langle E(p) x,-J y\rangle .
$$

Since $E(\sigma)$ and $J$ commute and $\widetilde{E}(\widetilde{\sigma})$ and $J$ commute, by assumption, we have (5.9). 


\section{THE SPECTRAL THEOREM FOR UNBOUNDED NORMAL OPERATORS BASED ON THE S-SPECTRUM}

In this section we will consider normal operators $T$ which are unbounded. The strategy will be to transform $T$ into a normal operator $Z_{T} \in \mathcal{B}(\mathcal{H})$ and use Theorem 4.7 and a change of variable argument to obtain a spectral theorem for $T$ based on the $S$-spectrum. Obtaining a spectral theorem for unbounded operators in the aforementioned way has been done in the classical case, i.e., when $\mathcal{H}$ is a complex Hilbert space, see, e.g., the book of Schmüdgen. ${ }^{33}$

Given $T \in \mathcal{L}(\mathcal{H})$, we let

$$
Z_{T}=T C_{T}^{1 / 2}
$$

where $C_{T}=\left(I_{\mathcal{H}}+T^{*} T\right)^{-1} \in \mathcal{B}(\mathcal{H})$ (the proof that $C_{T}$ is bounded can be carried out in a similar manner to the classical complex Hilbert case, see, e.g., Proposition 3.18(i) and Lemma 1.10 in Ref. 33).

Theorem 6.1. Let $T \in \mathcal{L}(\mathcal{H})$ be a densely defined closed operator on $\mathcal{H}$. The operator $Z_{T}$ has the following properties:

(i) $Z_{T} \in \mathcal{B}(\mathcal{H}),\left\|Z_{T}\right\| \leq 1$ and

$$
C_{T}=\left(I_{\mathcal{H}}+T^{*} T\right)^{-1}=I_{\mathcal{H}}-Z_{T}^{*} Z_{T}
$$

(ii) $\left(Z_{T}\right)^{*}=Z_{T^{*}}$

(iii) If $T$ is normal, then $Z_{T}$ is normal.

Proof. The proof is based on the proof of Lemma 5.7 in Ref. 33 and is broken into steps.

Step 1: Prove (i).

First note that

$$
\left\{C_{T} x: x \in \mathcal{H}\right\}=\mathcal{D}\left(I_{\mathcal{H}}+T^{*} T\right)=\mathcal{D}\left(T^{*} T\right) .
$$

Consequently, if $x \in \mathcal{H}$, then

$$
\begin{aligned}
\left\|T C_{T}^{1 / 2} C_{T}^{1 / 2} x\right\|^{2} & =\left\langle T^{*} T C_{T} x, C_{T} x\right\rangle \\
& \leq\left\langle\left(I_{\mathcal{H}}+T^{*} T\right) C_{T} x, C_{T} x\right\rangle \\
& =\left\langle C_{T}^{-1} C_{T} x, C_{T} x\right\rangle \\
& =\left\langle x, C_{T} x\right\rangle \\
& =\left\|C_{T}^{1 / 2} x\right\|^{2} .
\end{aligned}
$$

Thus, if $y \in\left\{C_{T}^{1 / 2} x: x \in \mathcal{H}\right\}$, then

$$
\left\|Z_{T} y\right\|=\left\|T C_{T}^{1 / 2} y\right\| \leq\|y\| .
$$

As $\operatorname{Ker}\left(C_{T}\right)=\{0\}$, we have that $\operatorname{Ker}\left(C_{T}^{1 / 2}\right)=\{0\}$ and thus $\left\{C_{T}^{1 / 2} x: x \in \mathcal{H}\right\}$ is a dense subset of $\mathcal{H}$. As $T$ is a closed operator by assumption and $C_{T}^{1 / 2} \in \mathcal{B}(\mathcal{H})$, we get that $Z_{T}$ is closed as well. Thus, we have $\left\{C_{T}^{1 / 2} x: x \in \mathcal{H}\right\} \subseteq \mathcal{D}(T), \mathcal{D}\left(Z_{T}\right)=\mathcal{H}$ and, in view of (6.4), $\left\|Z_{T}\right\| \leq 1$.

Next, it follows from (6.4) and $C^{1 / 2} T^{*} \subseteq Z_{T}^{*}$ that

$$
\begin{aligned}
\left(I_{\mathcal{H}}-C_{T}\right) C_{T}^{1 / 2} & =C_{T}^{1 / 2}\left(I_{\mathcal{H}}+T^{*} T\right) C_{T}-C_{T}^{1 / 2} C_{T} \\
& =C_{T}^{1 / 2} T^{*} T C_{T}^{1 / 2} C_{T}^{1 / 2} \\
& \subseteq Z_{T}^{*} Z_{T} C_{T}^{1 / 2} .
\end{aligned}
$$

Thus, $Z_{T}^{*} Z_{T} C_{T}^{1 / 2}=\left(I_{\mathcal{H}}-C_{T}\right) C_{T}^{1 / 2}$ and, as $\left\{C_{T}^{1 / 2} x: x \in \mathcal{H}\right\}$ is a dense subset of $\mathcal{H}$, we get (6.2). 
Step 2: Prove (ii).

Using (6.2) we get that $C_{T^{*}}=\left(I_{\mathcal{H}}+T T^{*}\right)^{-1}$. If $x \in \mathcal{D}\left(T^{*}\right)$, then let $y=C_{T^{*}} x$. Therefore,

$$
x=\left(I_{\mathcal{H}}+T T^{*}\right) y
$$

and

$$
T^{*} x=T^{*}\left(I_{\mathcal{H}}+T T^{*}\right) y=\left(I_{\mathcal{H}}+T^{*} T\right) T^{*} y .
$$

Thus, $C_{T^{*} X} \in \mathcal{D}\left(T^{*}\right)$ and hence

$$
C_{T} T^{*} x=T^{*} y=T^{*} C_{T^{*}} x .
$$

It follows easily from (6.5) and (6.2) that $p\left(C_{T^{*}}\right) x \in \mathcal{D}\left(T^{*}\right)$ and

$$
p\left(C_{T}\right) T^{*} x=T^{*} p\left(C_{T^{*}}\right) x
$$

for any real polynomial $p$ of a real variable. By the Weierstrass approximation theorem, there exists a sequence of real polynomials $\left\{\phi_{n}\right\}_{n=0}^{\infty}$ which converge uniformly in supremum norm to the function $t \mapsto t^{1 / 2}$ on $[0,1]$. Using Property (iv) of Theorem 3.11 we have that

$$
\lim _{n \uparrow \infty}\left\|\phi_{n}\left(C_{T}\right)-C_{T}^{1 / 2}\right\|=\lim _{n \uparrow \infty}\left\|\phi_{n}\left(C_{T^{*}}\right)-C_{T^{*}}^{1 / 2}\right\|=0 .
$$

Since $T$ is a closed operator, $T^{*}$ is also a closed operator. Thus, we have

$$
\begin{aligned}
C_{T}^{1 / 2} T^{*} x & =\lim _{n \uparrow \infty} \phi_{n}\left(C_{T}\right) T^{*} x=\lim _{n \uparrow \infty} T^{*} \phi_{n}\left(C_{T^{*}}\right) x \\
& =T^{*}\left(C_{T^{*}}\right)^{1 / 2} x \text { for } x \in \mathcal{D}\left(T^{*}\right) .
\end{aligned}
$$

As $C_{T}^{1 / 2} T^{*} \subseteq\left(T C_{T}^{1 / 2}\right)^{*}=Z_{T^{*}}$, we get that

$$
Z_{T^{*}} x=C_{T}^{1 / 2} T^{*} x=T^{*}\left(C_{T^{*}}\right)^{1 / 2} x=\left(Z_{T}\right)^{*} x
$$

for $x \in \mathcal{D}\left(T^{*}\right)$. Finally, since $\mathcal{D}\left(T^{*}\right)$ is dense in $\mathcal{H}$, we have that $Z_{T^{*} x}=\left(Z_{T}\right)^{*} x$, i.e., $Z_{T^{*}}=\left(Z_{T}\right)^{*}$.

Step 3: Prove (iii).

Using (6.2) on $T$ and $T^{*}$ and the fact that $T T^{*}=T^{*} T$ we have

$$
I_{\mathcal{H}}-Z_{T}^{*} Z_{T}=\left(I_{\mathcal{H}}+T^{*} T\right)^{-1}=\left(I_{\mathcal{H}}+T T^{*}\right)^{-1}=I_{\mathcal{H}}-Z_{T^{*}}^{*} Z_{T^{*}}
$$

Making use of Property (ii) we have that

$$
I_{\mathcal{H}}-Z_{T}^{*} Z_{T}=I_{\mathcal{H}}-Z_{T} Z_{T}^{*}
$$

i.e., $Z_{T}$ is normal.

We are now ready to state and prove a spectral theorem for unbounded normal operators on a quaternionic Hilbert space.

Theorem 6.2. Let $T$ be an unbounded right linear normal operator on $\mathcal{H}$ and $j \in \mathbb{S}$. There exists a uniquely determined spectral measure $E_{j}$ on $\Omega_{j}^{+}=\sigma_{S}(T) \cap \mathbb{C}_{j}^{+}$so that

$$
\langle T x, y\rangle=\int_{\Omega_{j}^{+}} \operatorname{Re}(p) d\left\langle E_{j}(p) x, y\right\rangle+\int_{\Omega_{j}^{+}} \operatorname{Im}(p) d\left\langle J E_{j}(p) x, y\right\rangle, \quad x \in \mathcal{D}(T),
$$

or, equivalently,

$$
\langle T x, y\rangle=\int_{\Omega_{j}^{+}} p d\left\langle\Pi_{+}^{j} E_{j}(p) x, y\right\rangle+\int_{\Omega_{j}^{+}} \bar{p} d\left\langle\Pi_{-}^{j} E_{j}(p) x, y\right\rangle, \quad x \in \mathcal{D}(T),
$$

where the operator $J \in \mathcal{B}(\mathcal{H})$ is operator appearing in the decomposition in Theorem 3.6 for $Z_{T} \in$ $\mathcal{B}(\mathcal{H})$ and $\Pi_{ \pm}^{j}$ are as in Theorem 4.7. Moreover, upon identifying the complex plane $\mathbb{C}_{k}$ with $\mathbb{C}_{j}$ in the natural way by the mapping $\varphi_{k j}$, we have $E_{j}\left(\varphi_{k j}(\sigma)\right)=E_{k}(\sigma), \sigma \in \mathfrak{B}\left(\Omega_{k}^{+}\right)$for all $j, k \in \mathbb{S}$.

Proof. The proof is broken into steps.

Step 1: Show that a spectral measure E exists so that (6.6) holds. 
Let $\mathbb{B}=\{p \in \mathbb{H}:|p|<1\}, \partial \mathbb{B}=\{p \in \mathbb{H}:|p|=1\}$, and $\overline{\mathbb{B}}=\mathbb{B} \cup \partial \mathbb{B}$. If $T$ is normal, then using Properties (i) and (iii) in Theorem 6.1 we get that $\left\|Z_{T}\right\| \leq 1$ and $Z_{T}$ is normal, respectively. Thus, we may use Theorem 4.7 to obtain a uniquely determined spectral measure $F$ on $\sigma_{S}\left(Z_{T}\right) \cap \mathbb{C}_{j}^{+}$so that

$$
f\left(Z_{T}\right)=\mathbb{I}(f)=\int_{\sigma_{S}\left(Z_{T}\right) \cap \mathbb{C}_{j}^{+}} f(p) d F(p) \quad \text { for } f \in \mathscr{C}_{0}\left(\sigma_{S}\left(Z_{T}\right) \cap \mathbb{C}_{j}^{+}, \mathbb{C}_{j}\right)
$$

when $\sigma_{S}(T) \cap \mathbb{R} \neq \emptyset$ and for all $f \in \mathscr{C}\left(\sigma_{S}\left(Z_{T}\right) \cap \mathbb{C}_{j}^{+}, \mathbb{C}_{j}\right)$ when $\sigma_{S}(T) \cap \mathbb{R}=\emptyset$. In addition, it follows from Theorem 3.2.6 in Ref. 18 that

$$
\sigma_{S}\left(Z_{T}\right) \subseteq\left\{p \in \mathbb{H}:|p| \leq\left\|Z_{T}\right\|\right\}
$$

and hence

$$
\sigma_{S}\left(Z_{T}\right) \cap \mathbb{C}_{j}^{+} \subseteq \overline{\mathbb{B}} \cap \mathbb{C}_{j}^{+}
$$

If $x \in \mathcal{H}$ and $\sigma \in \mathfrak{B}\left(\sigma_{S}(T) \cap \mathbb{C}_{j}^{+}\right)$, then, in view of item (v) in Lemma 5.3 and (6.8), we have

$$
\left\langle\left(I_{\mathcal{H}}-Z_{T}^{*} Z_{T}\right) F(\sigma) x, F(\sigma) x\right\rangle=\int_{\sigma}\left(1-|p|^{2}\right) d\langle F(p) x, x\rangle .
$$

Recall that $I_{\mathcal{H}}-Z_{T}^{*} Z_{T}=\left(I_{\mathcal{H}}+T^{*} T\right)^{-1}$, i.e., $\operatorname{Ker}\left(I_{\mathcal{H}}-Z_{T}^{*} Z_{T}\right)=\{0\}$. Thus, using (6.9) with

$$
\sigma=\partial \overline{\mathbb{B}} \cap \mathbb{C}_{j}^{+}
$$

we get that supp $F \subseteq \overline{\mathbb{B}} \cap \mathbb{C}_{j}^{+}$and $F\left(\partial \mathbb{B} \cap \mathbb{C}_{j}^{+}\right)=0$. Therefore,

$$
F\left(\mathbb{B} \cap \mathbb{C}_{j}^{+}\right)=F\left[\left(\overline{\mathbb{B}} \cap \mathbb{C}_{j}^{+}\right) \backslash \partial\left(\mathbb{B} \cap \mathbb{C}_{j}^{+}\right)\right]=I_{\mathcal{H}} .
$$

If $\varphi(p)=p\left(1-|p|^{2}\right)^{-1 / 2}$, then $\varphi \in \mathscr{B}_{\infty}\left(F, \sigma_{S}\left(Z_{T}\right) \cap \mathbb{C}_{j}^{+}, \mathbb{C}_{j}\right)$. In view of item (iii) and (v) of Theorem 5.11 we have

$$
\mathbb{I}(\varphi)=\mathbb{I}(f) \mathbb{I}(g),
$$

where

$$
f(p)=p \quad \text { and } \quad g(p)=\frac{1}{\sqrt{1-|p|^{2}}},
$$

and $\mathcal{D}(\mathbb{I}(\varphi))=\mathcal{D}(\mathbb{I}(g))$. Using Theorem 5.12, we have

$$
\mathbb{I}(g)=\mathbb{I}(1 / g)^{-1} .
$$

Consequently, we may use item (i) in Corollary 4.9 to obtain

$$
\mathbb{I}(g)=\left\{(\mathbb{I}(h))^{1 / 2}\right\}^{-1},
$$

where

$$
h(p)=1-|p|^{2} \in \mathscr{B}\left(F, \sigma_{S}\left(Z_{T}\right) \cap \mathbb{C}_{j}^{+}, \mathbb{C}_{j}\right) .
$$

Putting these observations together, we obtain

$$
\mathbb{I}(\varphi)=Z_{T}\left(C_{T}^{1 / 2}\right)^{-1} .
$$

Since $Z_{T}=T C_{T}^{1 / 2}$ we obtain $\varphi\left(Z_{T}\right) \subseteq T$. Using $C_{T}=\left(I_{\mathcal{H}}-Z_{T}^{*} Z_{T}\right)^{1 / 2}$, we get that $\mathbb{I}(\varphi) \subseteq T$. Thus, using Lemma 2.10 we get that

$$
\mathbb{I}(\varphi)=T
$$

Let $E_{j}(\sigma)=F\left(\varphi^{-1}(\sigma)\right)$, where

$$
\varphi^{-1}(\sigma)=\{p \in \mathbb{H}: \varphi(p) \in \sigma\} \quad \text { for } \quad \sigma \in \mathfrak{B}\left(\sigma_{S}(T) \cap \mathbb{C}_{j}^{+}\right) .
$$

It is readily checked that $E_{j}=F\left(\varphi^{-1}\right)$ defines a spectral measure on $\mathbb{C}_{j}^{+}$and thus using Lemma 5.13 we get (6.6). The equivalent assertion (6.7) is established in much the same way as the analogous assertion in Theorem 4.7. 
Step 2: Show that $E_{j}$ from Step 1 is unique.

If $E_{j}$ and $\widetilde{E}_{j}$ are spectral measures on $\sigma_{S}(T) \cap \mathbb{C}_{j}^{+}$which satisfy (6.6), then $F=E_{j}(\varphi)$ and $\widetilde{F}=$ $\widetilde{E}_{j}(\varphi)$ are both spectral measures so that

$$
\begin{aligned}
\left\langle Z_{T} x, y\right\rangle & =\int_{\overline{\mathbb{B}} \cap \mathbb{C}_{j}^{+}} \operatorname{Re}(p) d\langle F(p) x, y\rangle+\int_{\overline{\mathbb{B}} \cap \mathbb{C}_{j}^{+}} \operatorname{Im}(p) d\langle J F(p) x, y\rangle \\
& =\int_{\overline{\mathbb{B}} \cap \mathbb{C}_{j}^{+}} \operatorname{Re}(p) d\langle\widetilde{F}(p) x, y\rangle+\int_{\overline{\mathbb{B}} \cap \mathbb{C}_{j}^{+}} \operatorname{Im}(p) d\langle J \widetilde{F}(p) x, y\rangle, \quad x, y \in \mathcal{H} .
\end{aligned}
$$

Let $\mathscr{P}\left(\sigma_{S}\left(Z_{T}\right) \cap \mathbb{C}_{j}^{+}, \mathbb{R}\right)$ denote the space of real-valued polynomials on $\sigma_{S}\left(Z_{T}\right) \cap \mathbb{C}_{j}^{+}$. In view of Lemma 5.3 and Remark 5.4, (6.11) yields

$$
\begin{aligned}
\left\langle\psi\left(Z_{T}\right) x, x\right\rangle & =\int_{\sigma_{S}\left(Z_{T}\right) \cap \mathbb{C}_{j}^{+}} \psi(p) d\langle F(p) x, x\rangle \\
& =\int_{\sigma_{S}\left(Z_{T}\right) \cap \mathbb{C}_{j}^{+}} \psi(p) d\langle\widetilde{F}(p) x, x\rangle, \quad \psi \in \mathscr{P}\left(\sigma_{S}\left(Z_{T}\right) \cap \mathbb{C}_{j}^{+}, \mathbb{C}_{j}\right), \quad x \in \mathcal{H} .
\end{aligned}
$$

As $\mathscr{P}\left(\sigma_{S}\left(Z_{T}\right) \cap \mathbb{C}_{j}^{+}, \mathbb{C}_{j}\right)$ is a dense subset of $\mathscr{C}\left(\sigma_{S}\left(Z_{T}\right) \cap \mathbb{C}_{j}^{+}, \mathbb{C}_{j}\right)$ we have that

$$
\int_{\sigma_{S}\left(Z_{T}\right) \cap \mathbb{C}_{j}^{+}} \phi(p) d\langle F(p) x, x\rangle=\int_{\sigma_{S}\left(Z_{T}\right) \cap \mathbb{C}_{j}^{+}} \phi(p) d\langle\widetilde{F}(p) x, x\rangle
$$

for all $\phi \in \mathscr{C}\left(\sigma_{S}\left(Z_{T}\right) \cap \mathbb{C}_{j}^{+}, \mathbb{R}\right)$ and hence, in view of construction of the spectral measure given in Section IV, $F=\widetilde{F}$. Therefore, $E_{j}=\widetilde{E}_{j}$. The final assertion concerning $E_{j}$ and $E_{k}$ is proved in a similar manner to an analogous assertion in Theorem 4.7.

Corollary 6.3. In the setting of Theorem 6.2, the following statements hold:

(i) If $T \in \mathcal{L}(\mathcal{H})$ is a positive operator, then there exists a unique positive operator $W \in \mathcal{L}(\mathcal{H})$ so that $W^{2}=T$.

(ii) $\quad T \in \mathcal{L}(\mathcal{H})$ is self-adjoint if and only if

$$
\langle T x, y\rangle=\int_{\mathbb{R}} t d\langle E(t) x, y\rangle, \quad x \in \mathcal{D}(T), y \in \mathcal{H} .
$$

(iii) $\quad T \in \mathcal{L}(\mathcal{H})$ is anti self-adjoint if and only if

$$
\langle T x, y\rangle=\int_{[0, \infty)} t d\langle J E(t) x, y\rangle, \quad x \in \mathcal{D}(T), y \in \mathcal{H} .
$$

Proof. Using Theorem 6.2, the proof is completed as in Corollary 4.9.

Remark 6.4. We remind the reader that the functional calculus mentioned in Section $\mathrm{V}$ is applicable to unbounded normal operators $T \in \mathcal{L}(\mathcal{H})$. We conclude this section by stating, in the following corollary, such a functional calculus.

Corollary 6.5. Let $T, E_{j}$, and $J$ be as in Theorem 6.2. If $f, g \in \mathscr{B}_{\infty}\left(E, \sigma_{S}(T) \cap \mathbb{C}_{j}^{+}, \mathbb{C}_{j}\right)$ and $\alpha, \beta \in$ $\mathbb{C}_{j}$, then

(i) $\mathbb{I}(\bar{f})=\mathbb{I}(f)^{*}$.

(ii) $\mathbb{I}(\alpha f+\beta g)=\overline{\alpha \mathbb{I}(f)+\beta \mathbb{I}(g)}$.

(iii) $\mathbb{I}(f g)=\overline{\mathbb{I}(f) \mathbb{I}(g)}$.

(iv) $\mathbb{I}(f)$ is a closed normal operator on $\mathcal{H}$ and

$$
\mathbb{I}(f)^{*} \mathbb{I}(f)=\mathbb{I}(f \bar{f})=\mathbb{I}(\bar{f} f) .
$$

(v) $\mathcal{D}(\mathbb{I}(f) \mathbb{I}(g))=\mathcal{D}(\mathbb{I}(g)) \cap \mathcal{D}(\mathbb{I}(f g))$. 
(vi) If $x \in \mathcal{D}(\mathbb{I}(f))$ and $y \in \mathcal{D}(\mathbb{I}(g))$, then

$$
\langle\mathbb{I}(f) x, \mathbb{I}(g) y\rangle=\int_{\Omega} \operatorname{Re}(f(p) \overline{g(p)}) d\langle E(p) x, y\rangle+\int_{\Omega} \operatorname{Im}(f(p) \overline{g(p)}) d\langle J E(p) x, y\rangle .
$$

(vii) If $x \in \mathcal{D}(\mathbb{I}(f))$, then

$$
\|\mathbb{I}(f) x\|^{2}=\int_{\Omega}|f(p)|^{2} d\langle E(p) x, x\rangle .
$$

\section{ACKNOWLEDGMENTS}

D. Alpay thanks the Earl Katz family for endowing the chair which supported his research. D. P. Kimsey gratefully acknowledges the support of a Kreitman postdoctoral fellowship. F. Colombo acknowledges the Center for Advanced Studies of the Mathematical Department of the Ben-Gurion University of the Negev for the support and the kind hospitality during the period in which part of this paper has been written.

${ }^{1}$ Abu-Ghanem, K., Alpay, D., Colombo, F., Kimsey, D. P., and Sabadini, I., "Boundary interpolation for slice hyperholomorphic Schur functions," Integr. Equations Oper. Theory 82(2), 223-248 (2015).

2 Adler, S. L., "Quaternionic quantum mechanics and noncommutative dynamics," in Proceedings of the Second International A. D. Sakharov Conference on Physics, Moscow (World Scientific Publishing, River Edge, NJ, 1997), pp. 337-341.

${ }^{3}$ Alpay, D., The Schur Algorithm, Reproducing Kernel Spaces and System Theory, SMF/AMS Texts and Monographs Vol. 5 (American Mathematical Society, Providence, RI; Société Mathématique de France, Paris, 2001) (translated from the 1998 French original by S. S. Wilson).

${ }^{4}$ Alpay, D., Colombo, F., Gantner, J., and Kimsey, D. P., "Functions of the infinitesimal generator of a strongly continuous quaternionic group," Anal. Appl. (Singapore) (to appear).

5 Alpay, D., Colombo, F., Gantner, J., and Sabadini, I., “A new resolvent equation for the $S$-functional calculus," J. Geom. Anal. 25(3), 1939-1968 (2015).

${ }^{6}$ Alpay, D., Colombo, F., Kimsey, D. P., and Sabadini, I., "The spectral theorem for unitary operators based on the $S$ spectrum," Milan J. Math. (published online).

${ }^{7}$ Alpay, D., Colombo, F., Kimsey, D. P., and Sabadini, I., “An extension of Herglotz's theorem to the quaternions," J. Math. Anal. Appl. 421, 754-778 (2015).

${ }^{8}$ Alpay, D., Colombo, F., and Sabadini, I., "Schur functions and their realizations in the slice hyperholomorphic setting," Integr. Equations Oper. Theory 72(2), 253-289 (2012).

${ }^{9}$ Alpay, D., Colombo, F., and Sabadini, I., "Pontryagin-de Branges-Rovnyak spaces of slice hyperholomorphic functions," J. Anal. Math. 121, 87-125 (2013).

${ }^{10}$ Alpay, D., Colombo, F., and Sabadini, I., "Krein-Langer factorization and related topics in the slice hyperholomorphic setting," J. Geom. Anal. 24(2), 843-872 (2014).

${ }^{11}$ Alpay, D., Colombo, F., and Sabadini, I., "Perturbation of the generator of a quaternionic evolution operator," Anal. Appl. 13(4), 347-370 (2015)

12 Alpay, D., Dijksma, A., Rovnyak, J., and de Snoo, H., Schur Functions, Operator Colligations, and Reproducing Kernel Pontryagin Spaces, Operator Theory: Advances and Applications Vol. 96 (Birkhäuser Verlag, Basel, 1997).

${ }^{13}$ Alpay, D. and Shapiro, M., "Reproducing kernel quaternionic Pontryagin spaces," Integr. Equations Oper. Theory 50(4), 431-476 (2004).

${ }^{14}$ Birkhoff, G. and von Neumann, J., “The logic of quantum mechanics,” Ann. Math. 37(4), 823-843 (1936).

${ }^{15}$ Colombo, F. and Sabadini, I., "On some properties of the quaternionic functional calculus," J. Geom. Anal. 19(3), 601-627 (2009).

${ }^{16}$ Colombo, F. and Sabadini, I., "On the formulations of the quaternionic functional calculus," J. Geom. Phys. 60(10), 1490-1508 (2010).

${ }^{17}$ Colombo, F. and Sabadini, I., “The quaternionic evolution operator,” Adv. Math. 227(5), 1772-1805 (2011).

${ }^{18}$ Colombo, F., Sabadini, I., and Struppa, D. C., "Noncommutative functional calculus," in Progress in Mathematics, Theory and Applications of Slice Hyperholomorphic Functions Vol. 289 (Birkhäuser/Springer Basel AG, Basel, 2011).

${ }^{19}$ Dunford, N. and Schwartz, J. T., Linear Operators. Part II. Wiley Classics Library, Spectral Theory. Selfadjoint Operators in Hilbert Space (John Wiley \& Sons, Inc., New York, 1988), with the assistance of W. G. Bade and R. G. Bartle, Reprint of the 1963 original, A Wiley-Interscience Publication.

${ }^{20}$ Dunford, N. and Schwartz, J. T., Linear Operators General Theory. Part I. Wiley Classics Library (John Wiley \& Sons, Inc., New York, 1988), with the assistance of W. G. Bade and R. G. Bartle, Reprint of the 1958 original, A Wiley-Interscience Publication.

${ }^{21}$ Emch, G., "Mécanique quantique quaternionienne et relativité restreinte. I," Helv. Phys. Acta 36, 739-769 (1963).

${ }^{22}$ Farenick, D. R. and Pidkowich, B. A. F., "The spectral theorem in quaternions," Linear Algebra Appl. 371, 75-102 (2003)

${ }^{23}$ Finkelstein, D., Jauch, J. M., Schiminovich, S., and Speiser, D., "Foundations of quaternion quantum mechanics," J. Math. Phys. 3, 207-220 (1962). 
${ }^{24}$ Ghiloni, R., Moretti, V., and Perotti, A., “Continuous slice functional calculus in quaternionic Hilbert spaces,” Rev. Math. Phys. 25(4), 1350006 (2013).

${ }^{25}$ Ghiloni, R., Moretti, V., and Perotti, A., "Spectral properties of compact normal quaternionic operators," in Hypercomplex Analysis: New Perspectives and Applications, Trends in Mathematics, edited by Bernstein, S., Kähler, U., Sabadini, I., and Sommen, F. (Springer International Publishing, 2014) (English), pp. 133-143.

${ }^{26}$ Ghiloni, R. and Recupero, V., "Semigroups over real alternative *-algebras: Generation theorems and spherical sectorial operators," Trans. Amer. Math. Soc. (published online).

${ }^{27}$ Halmos, P. R., Measure Theory (D. Van Nostrand Company, Inc., New York, NY, 1950).

${ }^{28}$ Horwitz, L. P. and Biedenharn, L. C., "Quaternion quantum mechanics: Second quantization and gauge fields," Ann. Phys. 157(2), 432-488 (1984).

${ }^{29}$ Lax, P. D., Functional analysis, Pure and Applied Mathematics (Wiley-Interscience, John Wiley \& Sons, New York, 2002).

${ }^{30}$ Mitrea, M., Clifford Wavelets, Singular Integrals, and Hardy Spaces, Lecture Notes in Mathematics Vol. 1575 (SpringerVerlag, Berlin, 1994).

${ }^{31}$ Neumann, J. v., "Allgemeine eigenwerttheorie hermitescher funktionaloperatoren,” Math. Ann. 102(1), 49-131 (1930).

32 Royden, H. L. and Fitzpatrick, P. M., Real Analysis, 4th ed. (Macmillan Publishing Company, New York, 2010).

${ }^{33}$ Schmüdgen, K., Unbounded Self-Adjoint Operators on Hilbert space, Graduate Texts in Mathematics Vol. 265 (Springer, Dordrecht, 2012).

${ }^{34}$ Sharma, C. S. and Coulson, T. J., "Spectral theory for unitary operators on a quaternionic Hilbert space," J. Math. Phys. 28(9), 1941-1946 (1987).

35 Teichmüller, O., “Operatoren im Wachsschen Raum,” J. Reine Angew. Math. 1936(174), 73-124.

${ }^{36}$ Viswanath, K., "Normal operations on quaternionic Hilbert spaces," Trans. Am. Math. Soc. 162, 337-350 (1971). 